\title{
The Nonclinical Disposition and PK/PD Properties of GaINAc- conjugated siRNA Are Highly Predictable and Build Confidence in Translation to Man
}

Author list: Robin McDougall*, Diane Ramsden*, Sagar Agarwal, Saket Agarwal,

Krishna Aluri, Michael Arciprete, Christopher Brown, Elena Castellanos-Rizaldos, Klaus

Charisse, Saeho Chong, Joseph Cichocki, Kevin Fitzgerald, Varun Goel, Yongli Gu,

Dale Guenther, Bahru Habtemariam, Vasant Jadhav, Maja Janas, Muthusamy

Jayaraman, Jeffrey Kurz, Jing Li, Ju Liu, Xiumin Liu, Steven Liou, Chris Maclauchlin,

Martin Maier, Muthiah Manoharan, Jayaprakash K. Nair, Gabriel Robbie, Karyn

Schmidt, Peter Smith, Christopher Theile, Akshay Vaishnaw, Scott Waldron, Yuanxin

Xu, Xuemei Zhang, Ivan Zlatev, and Jing-Tao Wu

Alnylam Pharmaceuticals, Cambridge, Massachusetts (All Authors)

\section{FOOTNOTES}

* Robin McDougall and Diane Ramsden contributed equally to this work. 


\section{DMD-MR-2021-000428}

\section{Running Title:}

Predictable Cross-Species Translation of GalNAc-conjugated siRNA

\section{Corresponding Author:}

Jing-Tao Wu

Department: Drug Metabolism and Pharmacokinetics, Early Development; Company: Alnylam Pharmaceuticals Inc.

Address: 675 W Kendall St, Cambridge MA, 02142

Phone: (617) 704-4656

Email: jtwu@alnylam.com

Number of Text Pages: 54

Number of Tables: 7

Number of Figures: 10

Number of References: 38

Number of words in Abstract: 241

Number of words in Significant Statement: 54

Number of words in Introduction: 750

Number of words Discussion: 1626 


\section{DMD-MR-2021-000428}

\section{Abbreviations:}

$3 \mathrm{H}$, tritium; Ago2, protein argonaute-2; $\mathrm{AS}(\mathrm{N}-1) 3^{\prime}$ also designated as $3^{\prime} \mathrm{N}-1$, loss of a single nucleotide from the $3^{\prime}$ end of the antisense strand; ADME; absorption, distribution, metabolism and excretion; AS, antisense strand; ASGPR, asialglycoprotein receptor; AUC, area under the curve; BCRP, breast cancer resistance protein; BDC; bile duct cannulated; BSEP, bile salt export pump; $\mathrm{C}_{\max }$, maximum concentration; $\mathrm{Ct}$, threshold cycle; CYP, cytochrome P450; DNA, deoxyribonucleic acid; EDTA; ethylenediaminetetraacetic acid; ESC; enhanced stability chemistry; ESC+; enhanced stability chemistry with stabilizing modifications; FVC, femoral vein-cannulated; GalNAc, N-acetylgalactosamine; IS, internal standard; IV, intravenous; LC-HRMS, liquid chromatography coupled with high resolution mass spectrometry; LSC; liquid scintillation counting; MARG, microautoradiography; mRNA, messenger ribonucleic acid; PBST; phosphate buffer saline with triton X; PCR, polymerase chain reaction; PD, pharmacodynamics; P-gp, p-glycoprotein; PK, pharmacokinetics; PPB, plasma protein binding; QWBA, quantitative whole body autoradiography; RISC, RNA-induced silencing complex; RNAi, ribonucleic acid interference; RT-qPCR, real-time quantitative polymerase chain reaction; SC, subcutaneous; SD, standard deviation; siRNA, small interfering ribonucleic acid; siRNA1-siRNA22, 22 distinct siRNAs used in the in vitro and in vivo studies with data presented in this work; STC, standard template chemistry; $t_{1 / 2}$, half-life; $\mathrm{T}_{\max }$, time at which maximal concentration was observed. 


\section{DMD-MR-2021-000428}

\section{Abstract:}

Conjugation of oligonucleotide therapeutics, including small interfering ribonucleic acids (siRNAs) or antisense oligonucleotides (ASOs) to N-acetylgalactosamine (GalNAc) ligands has become the primary strategy for hepatocyte-targeted delivery, and with the recent approvals of GIVLAARI® (givosiran) for the treatment of acute hepatic porphyria, $\mathrm{OXLUMO}^{\mathrm{TM}}$ (lumasiran) for the treatment of primary hyperoxaluria, and Leqvio ${ }^{\circ}$ (inclisiran) for the treatment of hypercholesterolemia, the technology has been wellvalidated clinically. While much knowledge has been gained over decades of development there is a paucity of published literature on the DMPK properties of GalNAc-siRNA. With this in mind the goals of this mini-review are to provide an aggregate analysis of these nonclinical ADME data to build confidence on the translation of these properties to human. Upon subcutaneous administration, GalNAcconjugated siRNAs are quickly distributed to the liver, resulting in plasma pharmacokinetic (PK) properties that reflect rapid elimination through ASGPR-mediated uptake from circulation into hepatocytes. These studies confirm that liver PK, including half-life and, most importantly, siRNA levels in RNA-induced silencing complex (RISC) in hepatocytes are better predictors of pharmacodynamics (PD) than plasma PK. Several in vitro and in vivo nonclinical studies were conducted to characterize the absorption, distribution, metabolism and excretion (ADME) properties of GalNAcconjugated siRNAs. These studies demonstrate that the PK/PD and ADME properties of GalNAc-conjugated siRNAs are highly conserved across species, largely predictable, and can be accurately scaled to human, allowing us to identify efficacious and safe clinical dosing regimens in the absence of human liver PK profiles. 


\section{DMD-MR-2021-000428}

\section{Significance Statement:}

Several nonclinical ADME studies have been conducted in order to provide a comprehensive overview of the disposition and elimination of GalNAc-conjugated siRNAs and the PK/PD translation between species. These studies demonstrate that the ADME properties of GalNAc-conjugated siRNAs are well correlated and predictable across species building confidence in the ability to extrapolate to human. 


\section{DMD-MR-2021-000428}

\section{Introduction:}

RNA interference (RNAi) is an intrinsic mechanism of post-transcriptional gene silencing mediated by double stranded short oligonucleotides, 21-25 nucleotides in length, known as short interfering RNA (siRNA) (Fire et al., 1998; Meister \& Tuschl, 2004). This catalytic process begins with loading duplex siRNA into the RNA-induced silencing complex (RISC) and removal of passenger (sense) strand to generate functional RISC containing only the guide (antisense) strand. RISC loaded guide strands then bind to a complementary target mRNA through Watson-Crick base-pairing triggering the endonucleolytic cleavage of the target mRNA opposite the guide nucleotides at position 10-11 from the 5 ' end. After nearly two decades of research and development, ONPATTRO®, a partially modified siRNA encapsulated in a lipid nanoparticle (LNP), became the first RNAi therapeutic to receive marketing authorization in 2018.

Direct conjugation of the siRNA to a multivalent $\mathrm{N}$-acetylgalactosamine (GalNAc) ligand combined with extensive chemical modifications to stabilize the siRNA allowed for selective targeting of hepatocytes in the liver through the asialoglycoprotein receptor (ASGPR) and has been transformative for liver-directed therapeutic oligonucleotides (Nair et al., 2014). An early generation of GalNAc-conjugated siRNA, with only a few further modifications designated as standard template chemistry (STC), achieved clinical proof of concept but required a high and frequent dose regimen (Zimmermann et al., 2017). Subsequent design improvements, which include the substitution of the two terminal phosphodiester linkages at the antisense $3^{\prime}$ and $5^{\prime}$ ends and the sense strand $5^{\prime}$ end with phosphorothioate linkages led to the enhanced stabilization chemistry (ESC) design, with improved metabolic stability and potency enabling a reduction in total dose 


\section{DMD-MR-2021-000428}

amount required and allowing for less frequent administration (Nair et al., 2017). GIVLAARI@, the first GaINAc-conjugated siRNA with ESC design received regulatory approval in 2019. It is dosed at $2.5 \mathrm{mg} / \mathrm{kg}$ monthly by SC injection. Continued refinement of the chemical modification pattern led to the development of advanced ESC designs with increased metabolic stability (Foster et al., 2018), while inclusion of seed destabilizing modifications, like glycol nucleic acid (GNA), provides improved specificity, designated as ESC+ design (Janas et al., 2018). These advances have resulted in prolonged duration of target protein reduction in nonclinical studies and investigational clinical studies (Ray et al., 2020). Vutrisiran, an Advanced ESC GalNAcconjugated siRNA currently in Phase 3 clinical development, demonstrates sustained pharmacodynamic (PD) effect lasting up to 10 months following a single $25 \mathrm{mg} \mathrm{SC}$ dose in healthy volunteers (Habtemariam et al., 2020).

Unlike traditional small molecule drugs, siRNAs are large hydrophilic molecules. The two complementary strands of siRNA form double-helical structures of 19-21 base pairs with a molecular weight of $\sim 14,000 \mathrm{kDa}$. Their polyanionic backbone and hydrophilic character prevent passive uptake across cell membranes and therefore require specialized delivery solutions to achieve adequate cellular uptake. Over the past several years, knowledge of nonclinical ADME properties has been accumulated with dozens of GalNAc-conjugated siRNAs including STC, ESC, Advanced ESC and ESC+ designs. Data generated in nonclinical studies of GaINAc-conjugated siRNAs suggest that after SC administration, rapid hepatic uptake is followed by slow metabolism and clearance from the liver (Nair et al., 2017; Zimmermann et al., 2017). As such, plasma concentrations are transient and not directly reflective of the prolonged duration of PD. 


\section{DMD-MR-2021-000428}

The maximum plasma concentration after SC administration of GalNAc-conjugated siRNA in all nonclinical species was typically achieved around 0.25 hours to 2 hours post-dose, with the last detectable concentration in plasma, above the lower limit of quantification, observed between 4-12 hour, indicating rapid and efficient hepatic uptake (Foster et al., 2018; Nair et al., 2017). This was reproducible across different siRNAconjugate chemistries and various nonclinical species. In contrast peak liver concentrations in mice, rats and monkeys are generally observed between $2-8$ hours post SC administration which highlights the rapid and efficient uptake of GalNAc-siRNA. Recent work highlights an acidic intracellular depot that contributes significantly to the durable PD effect (Brown et al., 2020). This work demonstrates that the high metabolic stability of current generation GalNAc-conjugated siRNAs in intracellular compartments results in continuous and sustained release of functionally active siRNAs into cytoplasm, where they can load into RISC and elicit a prolonged PD effect.

The current series of nonclinical ADME investigations have been conducted over years to support the continuous development of GalNAc-siRNA. The goal of this works is to summarize the knowledge gained with respect to the absorption, distribution, metabolism and excretion (ADME) properties, to compare and contrast between molecules containing different sequences but similar chemical modifications, and to provide a reference to work we have completed aimed to describe the translation between nonclinical PK properties and human. 


\section{DMD-MR-2021-000428}

\section{MATERIALS \& METHODS}

\section{Chemicals and Reagents}

Clarity OTX lysis loading buffer and Clarity OTX 96-well solid phase extraction plates were obtained from Phenomenex (Torrance, CA). Optima liquid chromatography-mass spectrometry (LC-MS) grade acetonitrile (ACN) and water, 6X EMSA gel loading solution, SYBR gold nucleic acid gel stain were purchased from Thermo Scientific (Waltham, MA), and 1,1,1,3,3,3-hexafluoro-2-propranol (HFIPA), N,N-

diisopropylethylamine (DIEA), ammonium acetate, ammonium bicarbonate, and tetrahydrofuran were purchased from Millipore Sigma (Darmstadt, Germany). $\beta-\mathrm{N}$ acetylglucosaminadase, Nuclease P1, and glycobuffer 1 were purchased from New England Biolabs. 10\% Tris-Borate-EDTA (TBE) Gel (Bio-Rad Laboratories, CA, USA). Oligonucleotides were synthesized at Alnylam by solid-phase synthesis using an RNA synthesizer. Sterling solvents/reagents from Glen Research, 500-Å controlled pore glass (CPG) solid supports from Prime Synthesis, and 2'-OMe, 2'-F nucleoside 3'phosphoramidites from Hongene were all used as received. Low-water content acetonitrile was purchased from EMD Chemicals. Male hepatocytes were purchased from BiolVT (Westbury, NY). All siRNAs had high-performance liquid chromatography (HPLC) purity of $>90 \%$ and all tritium-labeled siRNAs had HPLC radiopurity of $>96 \%$ and a tritium specific activity of $>250 \mu \mathrm{Ci}$ per mg of siRNA. The tritium specific activity was measured using a Hidex 300 SL liquid scintillation counter (Lablogic).

\section{GaINAc-siRNA Synthesis and Selection Criteria for Inclusion in Specific Studies}

Synthesis of GalNAc-siRNAs used in this analysis was conducted as previously described (Nair et al., 2014). The general chemical modifications previously disclosed 


\section{DMD-MR-2021-000428}

for late stage development siRNA including: revusiran, givosiran and lumasiran were utilized in all GalNAc-siRNAs described throughout this report (Shen \& Corey, 2018) and are depicted in Figure 1, in cases where glycol nucleic acid was used, the design was consistent with those published (Schlegel et al., 2017). For assessment of sex dependent differences in GalNAc-siRNA PK, all available data obtained in male and female animals was `included in the analysis. For mechanistic studies including in vitro confirmation of duplex metabolism, siRNA2 was used as the surrogate GalNAc-siRNA as it is reflective of the ESC design and has a complete data package available (including radiolabeled ADME, QWBA, and metabolite assessment including determination of metabolite contributions to pharmacology). All data available in the $5 / 6$ nephrectomy model and rat ADME/QWBA studies is included. The siRNA used in these studies was decided based on the stage of individual projects and project needs to inform their development. All studies describe data generated with GalNAc-siRNAs which were randomly anonymized through serial numbering from 1-to-22. There is no data presented for siRNA10.

\section{In Vitro Metabolism of GalNAc-conjugated siRNA}

siRNA2 was incubated at $160 \mu \mathrm{g} / \mathrm{mL}$ with $\beta$-N-acetylglucosaminadase at $37^{\circ} \mathrm{C}$ for 0,1 and $2 \mathrm{~h}$ in glycobuffer 1 . siRNA8 was incubated at $10 \mu \mathrm{g} / \mathrm{mL}$ with plated rat, monkey and human hepatocytes. Samples were removed at designated time-points and pooled for LC-MS analysis. Reactions were terminated by the addition of EDTA solution, frozen in liquid nitrogen followed by lysis at room temperature in the presence of lysis-loading buffer. The resulting samples were extracted on Clarity OTX SPE, according to 


\section{DMD-MR-2021-000428}

manufacturer's instructions, and concentrations of antisense strand and sense strand were quantified by LC-MS.

\section{In Vitro Metabolism of siRNA}

siRNA2 duplex and single strands (antisense and sense strand) separately were incubated (16 and $6 \mu \mathrm{g} / \mathrm{mL}$ ) with single strand specific nucleases including Nuclease P1. This nuclease acts on single strands from the 3' direction. Enzyme incubations were conducted at three activity levels each, ranging from 0.2 to 400 units. Aliquots were removed for analysis after 15- and 60-min. Samples were analyzed by liquid chromatography-high resolution mass spectrometry (LC-HRMS) and were normalized to the matrix only (containing no enzyme) control values. Electrophoretic mobility shift assays (EMSA) were used to determine if complementary antisense and sense strand, comprising siRNA2 or siRNA19, mixed at equivalent concentrations $(8 \mu \mathrm{g} / \mathrm{mL}$ or 4.8 $\mu \mathrm{g} / \mathrm{mL}$ ), would form duplex in PBS and $99 \%$ human plasma.

\section{Nonclinical in vivo PKPD studies}

All animal studies were conducted in accordance with local, state, and federal regulations as applicable and were approved by the Institutional Animal Care and Use Committee (IACUC). Studies were conducted in male and female Sprague Dawley rats, male and female CD1 and C57BL/6 and male and female cynomolgus monkeys from various $\mathrm{CROS}$ (i.e. Charles river labs, Covance). Typically samples from three animals per group were used to describe plasma, tissue and excretion (urine and feces) pharmacokinetic properties. For studies in mice and rats composite profiles were obtained due to the large sample volumes needed for quantitation of siRNA levels. Studies in rats were conducted using animals at least 8 weeks of age with a target 
DMD-MR-2021-000428

weight of 300 to $400 \mathrm{~g}$ at the initiation of dosing. Rodents were group housed with up to three animals of the same sex and same dosing group together, with the exception of animals identified for urine collection that were housed individually in metabolism cages for the duration of the urine collection period. All animals were provided enrichment activities. Chow was provided ad libitum, except during designated procedures and water was freely available via automatic watering systems. Terminal blood samples were collected via jugular venipuncture or abdominal aorta (at least $0.5 \mathrm{~mL}$ ) at designated time-points into $\mathrm{K}_{2}$ EDTA containing tubes for preparation of plasma. For studies in monkeys, naïve cynomolgus (Macaca Fascicularis) originating from China that weighed between 2-4 kg with the target age of 2 to 4 years were used. Monkeys were socially housed (up to three animals of the same sex and dosing group together), except for directly following procedures or when used for collection of urine, enrichment activities were provided, food was offered twice daily and water was available ad libitum. Monkeys were used as the pharmacologically relevant species with more relevant target phenotyping comparable to human. For pharmacodynamic analysis up to $3 \mathrm{~mL}$ blood was collected at timepoints spanning Day 1 up to Day 99. At designated time-points (i.e. $0.25,0.5,1,2,4,8,12$ and 24 h post-dose) target $1 \mathrm{~mL}$ of blood was removed via venipuncture into $\mathrm{K}_{2}$ EDTA tubes for preparation of plasma to support PK parameter estimation and metabolite profiling. To support derivation of liver PK parameters, liver biopsies (target $150-200 \mathrm{mg} /$ biopsy) were removed from a single monkey per group per time-point for quantitation of siRNA levels and up to three biopsies were possible per animal. Samples were stored at $-70^{\circ} \mathrm{C}$ until analysis. Studies in rat and monkey were conducted using various dose levels spanning from 


\section{DMD-MR-2021-000428}

$0.03 \mathrm{mg} / \mathrm{kg}$ to $30 \mathrm{mg} / \mathrm{kg}$ given as an intravenous (iv) bolus injection, or infusion into a lateral tail vein, or through subcutaneous administration into the scapular or mid-dorsal areas. Historical knowledge related to the safety of this modality and the potency of PD observed during screening assays was used to design dose levels and regimens. Dose volume was limited to $1.5 \mathrm{~mL} / \mathrm{kg}$. Where comparisons were made between groups to evaluate the statistical significance a two-tailed, unpaired, student t-test with unequal variance was used.

\section{Assessing the Role of Renal Clearance using 5/6 Nephrectomy Studies}

5/6 nephrectomy and sham male Sprague Dawley rats were prepared as described previously (Kliem et al., 1996). In short, $2 / 3$ of one kidney were removed followed by a second surgery (3-7 days later) to remove the entire second kidney. Renal damage was confirmed by monitoring urinary excretion of protein, creatinine clearance and histopathological examination. siRNA5, siRNA11 and siRNA14 were dosed approximately 4 weeks after sham or $5 / 6$ nephrectomy surgery data from three to five rats per time-point were combined for generation of composite PK profiles. Blood samples were typically collected $(0.3 \mathrm{~mL})$ at pre-dose, $0.5,2,4,8,24$ and 336 hours for preparation of plasma and generation of plasma PK parameters. Liver and kidney samples were collected at similar time-points. Urine and feces were collected at timeintervals including, 0-4, 4-8, 8-24, 24-48, 48-72 hours for siRNA quantitation. Student T-tests were used to evaluate whether any differences between sham operated and $5 / 6$ nephrectomized rats were significantly significant. The following parameters were assessed: daily clinical observations, body weight, clinical pathology (clinical chemistry), creatinine, and urine collection for chemistry and biomarkers. At the end of the 


\section{DMD-MR-2021-000428}

treatment period on Days 2, 4, and 8, the animals were euthanized, and a complete macroscopic examination was performed. Liver and kidneys were weighed, and microscopic evaluation was conducted. A section of the liver (right lateral lobe) and one half of the remaining kidney in the nephrectomized animals and half of the left kidney in the sham animals was frozen for test article concentration analysis.

\section{Mass Balance and Excretion Studies in Rats}

Tritiated siRNA was prepared as described in detail in the Supplemental Material. Male bile duct-cannulated (BDC) and femoral vein-cannulated (FVC) Sprague Dawley rats were utilized. Dose levels of $3 \mathrm{mg} / \mathrm{kg}$ were selected for siRNA2 and siRNA6 and 10 $\mathrm{mg} / \mathrm{kg}$ for siRNA20. The following samples were collected for analysis of excretion of radioactivity and mass balance. Urine samples were collected on dry ice at predose (overnight), and 0-6, 6-12, 12-24 hours (h) postdose, and at 24-h intervals through $1344 \mathrm{~h}$ postdose. Feces samples were collected on dry ice predose (overnight) and at 0$6,6-12,12-24 \mathrm{~h}$ postdose, and at 24-h intervals through $1344 \mathrm{~h}$ postdose. After each 24-h excreta collection through $1344 \mathrm{~h}$ postdose, with the exception of cage change days, cages were rinsed with water followed by an approximate $30-\mathrm{mL}$ ethanol:deionized water (v:v, 1:1) rinse. Cage rinse samples were collected. At the time of each cage change and after the last excreta collection, cages were washed and wiped with at least $90 \mathrm{~mL}$ of Count-Off solution (equivalent to RadiacWash). The cage wash and cage wipe samples were collected for LSC analysis. In addition to the fecal and urine samples in the bile duct cannulated rats bile was also sampled for determining mass balance and excretion pathways. Bile samples were collected on dry ice from each animal at predose (overnight), at 0-6,6-12, 12-24 h postdose, and at 24-h. 


\section{DMD-MR-2021-000428}

Ultima Gold XR scintillation cocktail was used for samples analyzed directly. All samples were analyzed for radioactivity in Model2900TR or 2910TRliquid scintillation counters (Packard Instrument Company) for at least 5 minutes or 100,000 counts. Each sample was homogenized before radioanalysis (unless the entire sample was used for analysis). All samples were analyzed in triplicate (as applicable) if sample size allowed. Scintillation counting data (cpm) were automatically corrected for counting efficiency using the external standardization technique and an instrument-stored quench curve generated from a series of sealed quenched standards. A second set of duplicate or triplicate (as applicable) aliquots of bile, blood, plasma and urine were dried and analyzed. The results were compared to original analysis results to determine the tritiated water content of each sample.

\section{Quantitative Whole-body Autoradiography Studies in Rats}

Male Sprague Dawley rats bile duct-cannulated (BDC) and intact femoral veincannulated (FVC) were utilized. Dose levels of $3 \mathrm{mg} / \mathrm{kg}$ were selected for siRNA2 and siRNA6 and $10 \mathrm{mg} / \mathrm{kg}$ for siRNA20. The total amount of radioactivity administered was approximately $500 \mu \mathrm{Ci} / \mathrm{kg}$. As much blood as possible was collected from all animals via exsanguination (cardiac puncture). Blood was transferred into tubes to obtain plasma within 30 minutes of collection. One animal/time point was prepared for QWBA at staggered time-points through 1344 hours (56 days) post-dose. The carcasses were immediately frozen, and each carcass stored at approximately $-70^{\circ} \mathrm{C}$. The pinna, limbs, hair (as necessary), and tail, of each frozen carcass for QWBA were removed and frozen into a block, and a section set from each animal was prepared to be exposed on phosphorimaging screens for seven days. Specified tissues, organs, and fluids were 


\section{DMD-MR-2021-000428}

analyzed. Tissue concentrations were interpolated from each standard curve as nanocuries/g and then converted to $n g$ equivalents/g on the basis of the test article specific activity. Tissue concentration data was determined if examination of the autoradiographs warranted analysis. Specified tissues, organs, and fluids were analyzed, and the tissue concentrations were interpolated from each standard curve as microcuries or nanocuries per gram $(\mu \mathrm{Ci} / \mathrm{g}$ or $\mathrm{nCi} / \mathrm{g})$.

\section{Red Blood cell partitioning in rat}

Blood was mixed by inverting several times. Triplicate weighed aliquots of each blood sample (approximately $0.1 \mathrm{~g}$ ) were removed for radioanalysis by LSC. A sufficient amount of commercial solubilizing agent was added to digest each sample. Samples were incubated for at least 1 hour at approximately $60^{\circ} \mathrm{C}$. The samples were allowed to sit at least overnight to allow any foaming to dissipate. Ultima Gold XR scintillation cocktail was added, and the samples were shaken and analyzed by LSC. Additional duplicate weighed aliquots of blood were dried under a stream of nitrogen and reconstituted in water. A sufficient amount of commercial solubilizing agent was added to digest each sample. Samples were incubated for at least 1 hour at approximately $60^{\circ} \mathrm{C}$. The samples were allowed to sit at least overnight to allow any foaming to dissipate. Ultima Gold XR scintillation cocktail was added and the samples were shaken and analyzed by LSC. The remaining blood samples were centrifuged within 30 minutes of collection to separate plasma at 3000 -rpm $(1500 \times \mathrm{g})$ for approximately 10 minutes at approximately $5^{\circ} \mathrm{C}$. The resulting plasma was mixed and duplicate weighed aliquots of each resultant plasma sample (approximately $0.1 \mathrm{~g}$, as available) were removed for radioanalysis by LSC. 


\section{DMD-MR-2021-000428}

\section{Microautoradiography evaluation}

A set of representative sections for each male rat (with internal standards for each section) were mounted on thin cardboard supports. The mounted sections were exposed to phosphorimaging screens. Remaining dose formulation spiked blood calibration standards at 8 different concentrations were co-exposed with all sections and the images were used for calibrating the image analysis software. The exposed screens were scanned using a Typhoon FLA 9500 Phosphor Imager and data was acquired as DensRFU/ $\mu \mathrm{m}^{\wedge} 2$. The autoradiographic standard image data (calibration and internal standards) was sampled using $\mathrm{MCID}^{\mathrm{TM}}$ software to create a calibrated standard curve and to verify section thickness uniformity. Specified tissues, organs, and fluids were analyzed and the tissue concentrations were interpolated from each standard curve as microcuries per gram ( $\mu \mathrm{Ci} / \mathrm{g}$ or $\mathrm{nCi} / \mathrm{g})$. The concentrations were converted to ng equivalents of parent compound per gram of tissue based on the specific activity of the parent compound in the dosing formulation.

\section{Evaluation of the Potential for Enterohepatic Recirculation to Impact}

\section{Pharmacodynamics}

Intact and bile duct cannulated female Sprague Dawley rats $(n=4 /$ group) were dosed with $1 \mathrm{mg} / \mathrm{kg}$ siRNA11 followed by serial blood collections $(0.3 \mathrm{~mL})$ at pre-dose, 24,48 , $72,96,120,144$ and $168 \mathrm{~h}$ post dose. Bile and liver samples were also collected for potential analysis. Serum was prepared from whole blood for evaluation of the target present using a commercially available ELISA kit. Female rats were chosen because previous studies using siRNA11, at this dose level, demonstrated recovery of pharmacodynamic response within the time-frame applied in this study. 


\section{DMD-MR-2021-000428}

\section{In Vitro Investigation on the Potential for Truncation Metabolites to Contribute to Pharmacodynamics}

For each duplex evaluated, the antisense strand was truncated from either the $5^{\prime}$ or $3^{\prime}$ end, relative to the full antisense strand of siRNA2. Because the antisense strand is the active strand, the composition of the sense strand was not changed. As such, all duplexes were annealed to the full-length sense strand, the same sense strand employed in siRNA2 drug substance. Hep3B cells were transfected by adding $4.9 \mu \mathrm{L}$ of Optimized Minimal Essential Medium (Opti-MEM) plus $0.1 \mu \mathrm{L}$ of Lipofectamine® RNAiMAX Transfection Reagent per well (Invitrogen [Carlsbad, CA] Catalog No. $13778150)$ to $5 \mu \mathrm{L}$ of siRNA duplexes per well into a 384-well plate. The plate was incubated at room temperature for 15 minutes, and then $40 \mu \mathrm{L}$ of Eagle's Minimum Essential Medium (EMEM) containing $\sim 5 \times 10^{3}$ cells was added to the siRNA mixture. Cells were incubated for 24 hours before RNA purification. Two separate experiments were performed at final duplex concentrations of 0.0016 and $0.16 \mu \mathrm{g} / \mathrm{mL}$, respectively, and messenger RNA (mRNA) was assayed 24 hours after transfection by quantitative polymerase chain reaction (qPCR). Briefly, $2 \mu \mathrm{L}$ of cDNA was added to a master mix containing $0.5 \mu \mathrm{L}$ of GAPDH TaqMan® Probe (Hs99999905), $0.5 \mu \mathrm{L}$ of commercially available target probe, and $5 \mu \mathrm{L}$ of LightCycler ${ }^{\circledR} 480$ Probes Master (Roche [Indianapolis, IN] Catalog No. 04887301001) per well in a 384-well plate (Roche Catalog No. 04887301001). qPCR was done in a LightCycler® 480 qPCR system (Roche) using the Delta-Delta Threshold Cycle (Relative Quantitation) [ $\triangle \Delta \mathrm{CT}(\mathrm{RQ})]$ assay. Each duplex was tested in 4 independent transfections. To calculate relative fold change, data were analyzed using the ${ }^{\Delta \Lambda} C T(R Q)$ method and normalized to assays 


\section{DMD-MR-2021-000428}

performed with cells transfected with 10-nM non-targeting siRNA (ie, mock transfected cells). Data are expressed as percent of message remaining, and error is expressed as standard deviation (SD), derived from the 4 transfection replicates.

\section{Mass-spectroscopic (LC-HRMS) Analysis}

Identities and purities of oligonucleotides were confirmed by electrospray ionization mass spectroscopy and ion exchange high-performance liquid chromatography. The LC-TOF-MS assays quantified the concentrations of specific GalNAc-conjugated siRNAs by detecting the antisense strand and sense strand portions of the duplex. Samples were spiked with internal standard (IS), processed by solid phase extraction, and analyzed using reversed-phase ultra-high-performance LC with Turbo lon Spray TOF-MS detection. Accurate masses of 10 ions for each strand of the analyte, sense, and antisense and each strand of the IS, sense and antisense, were monitored in the negative ionization mode. The peak area for the analyte or IS was the sum of the response from the respective 10 ions. The peak area ratios of the respective analytesense/IS-sense and analyte-antisense/IS-antisense single strands were used. The detection limit for LC-HRMS detection was typically $10 \mathrm{ng} / \mathrm{mL}$ or $100 \mathrm{ng} / \mathrm{g}$ for plasma and tissue levels respectively. Prior to using analytical methods, to support these nonclinical investigations, the methods were validated or qualified according to the recommendations set forth by regulatory bodies. Standard methods, instrumentation, columns and IS were used as previously published (J. Li et al., 2019; Liu et al., 2019).

\section{Quantification of siRNA SL-RT-qPCR}

Quantitation of siRNA concentrations was conducted by diluting samples with equivalent volume of PBST (0.25\% Triton X-100), or in the case of tissue samples 


\section{DMD-MR-2021-000428}

reconstituting $10 \mathrm{mg}$ of powdered tissue in $1 \mathrm{~mL}$ of PBST. Diluted samples were incubated at $95^{\circ} \mathrm{C}$ for $10 \mathrm{~min}$, vortexed and immediately placed on ice for $10 \mathrm{~min}$, then centrifuged for $10 \mathrm{~min}$ at 16,000 at $4^{\circ} \mathrm{C}$. Supernatants were transferred to DNAse/RNase free tubes and analyzed immediately or frozen until analysis. Prior to stem-loop PCR, described below, a minimum of $20 \mu \mathrm{L}$ of sample (plasma or tissue) was transferred into a 96 -well plate and heated at $95^{\circ} \mathrm{C}$ for 10 minutes, to allow duplex denaturing removal of secondary structures.

The general strategies previously published were employed to design primers and probes using a TaqMan ${ }^{\text {TM }}$-based approach (Castellanos-Rizaldos et al., 2020; Chen et al., 2005; Landesman et al., 2010). In short the TaqMan MicroRNA reverse transcription kit was used according to the manufacturer's recommendations under the following conditions; $5 \mu \mathrm{L}$ of the denatured sample/standard/QC was added to $10 \mu \mathrm{L}$ of the $\mathrm{RT}$ reaction mixture containing $1 \mathrm{x}$ buffer, $1 \mathrm{x}$ reverse transcriptase, RNAse inhibitor, stemloop oligonucleotide, water and dNTPs Reactions were incubated at $16{ }^{\circ} \mathrm{C}$ for $30 \mathrm{~min}$, followed by $42^{\circ} \mathrm{C}$ for $30 \mathrm{in}$, and inactivated at $85^{\circ} \mathrm{C}$ for $5 \mathrm{~min}$. QPCR was performed on a ViiA 7-Real-Time PCR System using a 384-well block and TaqMan Fast advanced master mix following the manufacturer's protocols.

\section{Quantification of RISC Loaded siRNA}

RISC-loaded siRNA (antisense strand) quantification using stem-loop RT-qPCR following Ago2 immunoprecipitation from liver samples was conducted as reported previously (Castellanos-Rizaldos et al., 2020). Processed liver samples were resuspended to $100 \mathrm{mg} / \mathrm{mL}$ in prechilled lysis buffer (50 mM Tris- $\mathrm{HCl}, \mathrm{pH} 7.5,150 \mathrm{mM}$ $\mathrm{NaCl}, 2 \mathrm{mM}$ EDTA, 0.5\% Triton-X 100, supplemented with one tablet of cOmplete TM 


\section{DMD-MR-2021-000428}

Mini, EDTA-free protease inhibitor cocktail and $1 \mathrm{mM}$ of phenylmehanesulfonyl fluoride. Samples were precleared using QAE-Sephadex R A-50 resin and subjected to Ago2 immunoprecipitation by incubation with anti-mouse Ago2 and normal mouse IgG as a control using Protein G Dynabeads (ThermoFisher Scientific). RISC-loaded siRNA was subsequently quantified by the stem-loop RT-qPCR approach described above.

\section{Absolute Quantitation and Metabolite Profiling from Cell Lysate, Plasma and}

\section{Tissue Samples}

Absolute quantitation of concentrations of specific siRNA in cell lysates, plasma, tissue, urine and fecal samples and metabolite profiling were performed using a Dionex liquid chromatography system coupled to Q Exactive mass spectrometer (Thermo scientific Waltham MA). Solid phase extraction using Clarity OTX 96 well plates was used to cleanup samples before LC-MS analysis (Rudge et al., 2011). 1\% HFIPA/0.1\% DIEA with $10 \mu \mathrm{M}$ EDTA in water and $0.075 \%$ HFIPA/0.0375\% DIEA with $10 \mu \mathrm{M}$ EDTA in 65/35 ACN/water were used as mobile phase A and B, respectively. LC-MS analysis for siRNA was performed either using $\mathrm{t}$-sim or MS/MS mode and utilized a gradient reaching $40 \%$ B in 4 minutes or up to $20 \%$ B in 20 minutes for quantitation and metabolite profiling, respectively. Quantitation of parent siRNA (full length antisense strand) and the potential major antisense metabolite $A S\left(3^{\prime} \mathrm{N}-1\right)$ was conducted using Thermo Quan browser with a calibration curve ranging from 10-5000 $\mathrm{ng} / \mathrm{mL}$ and metabolite profiling of siRNA was performed using ProMass (Hail et al., 2004) (Novatia, Newtown, PA). Relative percentages of metabolites were calculated using peak intensities relative to total intensity within the sample. Metabolites were confirmed by manual evaluation of MS2 when available. 


\section{DMD-MR-2021-000428}

\section{mRNA Quantitation for Pharmacodynamic Evaluations}

Total mRNA was isolated, and reverse transcribed into cDNA as previously described (Foster et al., 2018). qPCR reactions were performed using gene specific TaqMan assays for each target gene of interest against representative species.

\section{PK and PD Analysis}

Pharmacokinetic parameters were calculated using noncompartmental analysis in Phoenix WinNonlin 7.0. Pharmacodynamics were evaluated by measuring target mRNA levels in liver samples or protein levels in serum using qPCR or ELISA as previously described (Foster et al., 2018). 


\section{DMD-MR-2021-000428}

\section{RESULTS:}

\section{GaINAc-conjugated siRNAs retain their duplex structure in circulation}

The direct determination of double stranded vs. single stranded siRNAs in biological matrices using LC-HRMS is technically challenging as typical experimental conditions employed during LC-HRMS analysis would generally denature a double stranded siRNA into its single strands. Thus, a series of experiments was conducted to evaluate whether the two strands stayed as a duplex in circulation and whether the concentration of one strand would represent the other strand as well as the duplex. Initially, Nuclease P1, a mixture of nucleases known to degrade RNA and DNA were incubated with single strands and/or duplex siRNA. As depicted in Supplemental Table 1, the duplex was stable under all test conditions while single strands (both sense and antisense) were quickly metabolized by Nuclease P1. This in vitro experiment suggested that the sense and antisense strands are likely not stable in circulation unless present as a duplex. Further in vivo experiments confirmed that both sense and antisense strand concentrations were typically represented at a 1:1 ratio across rat, monkey and human plasma over extended periods of time (Figure 2A, 2B). The plasma pharmacokinetic parameters and the corresponding figures demonstrate no significant differences in derived parameters, as determined with a 2-tailed, unpaired, student t-test with unequal variance. At similar dose levels the plasma $C_{\max }(\mathrm{ng} / \mathrm{mL})$ are consistent across species (Figure 2A) but the AUC $\left(h^{*} n g / m L\right)$ in human is higher than what is observed in rat and monkey (Figure 2A, 2B). Comparison of exposures between siRNA1 and siRNA5 demonstrates that the exposure is consistent between these GalNAc-siRNAs. siRNA1 represents a newer chemistry containing a GNA while siRNA5 is an advanced ESC 


\section{DMD-MR-2021-000428}

GalNAc-siRNA. In addition, separate EMSA studies show that both siRNA2 and siRNA19 would form duplexes in PBS and/or plasma (siRNA19 only) when sense and antisense strands were combined at equivalent ratios (Supplemental Figure 1). Taken together, these data strongly suggest that GalNAc-conjugated siRNAs stay as a duplex in circulation and the measured levels of antisense strand are representative of intact duplex.

\section{Absorption}

An evaluation of sex dependence on plasma PK parameters was performed following dosing of 5 and 7 different GalNAc-conjugated siRNAs in rats or cynomolgus monkeys, respectively (Table 1). The results demonstrated comparable $C_{\max }(\mathrm{ng} / \mathrm{mL}), A \cup C_{\text {last }}$ ( $\left.h^{*} n g / m L\right), T_{\max }(h), T_{1 / 2}(h)$ and \% of dose observed in plasma at $C_{\max }$ between male and female of both rats and monkeys with no statistically significant difference as measured using a 2-tailed, unpaired, student t-test with unequal variance (Table 1). The plasma PK in rats, monkeys and humans increased in a dose proportional manner at doses up to $10 \mathrm{mg} / \mathrm{kg}$ and are well correlated confirming that human plasma PK can be predicted through allometric scaling (Figure 2C $-2 \mathrm{D}$ ). Dose levels $>10 \mathrm{mg} / \mathrm{kg}$ begin to show greater than dose proportional increases as exemplified by comparing the AUC across increasing dose levels; human (Figure 2E) and monkey (Figure 2F). This is likely due to transient saturation of ASGPR-mediated uptake clearance which leads to increased plasma exposure. Following SC injection, GalNAc-conjugated siRNA is absorbed quickly from the injection site and subject to rapid clearance from plasma (dominated by ASGPR-mediated uptake into the liver) with plasma $\mathrm{C}_{\max }$ typically observed between 0.2 - 12 hours and plasma half-life less than 5 hours in rat, 10 hours 
DMD-MR-2021-000428

in monkey and human (Table 1 and Figure $2 A-B$ ). At plasma $T_{\max }$, less than $1 \%$ of the SC injected dose resides in the plasma (scaling plasma concentration by volume), Table 1. There are differences between the plasma PK profile when comparing IV and SC dosing (Figure 2G), which leads to higher initial plasma concentrations following IV dosing. These circulating concentrations can lead to saturation of ASGPR-mediated uptake clearance thereby complicating the interpretation of systemic bioavailability.

Designing experiments to determine the absolute systemic bioavailability is complicated by a desire to use a low enough dose to avoid ASGPR saturation while still dosing high enough to enable adequate plasma exposure to accurately characterize the AUC. Due to rapid ASGPR-mediated distribution into the liver, the plasma exposure of GalNAcconjugated siRNA is limited and thus requires relatively high dose levels to ensure detection long enough to characterize plasma PK parameters. When high doses are administered via IV bolus injection, the systemic exposure reaches levels that saturate the ASGPR uptake capacity for a period of time after injection, which subsequently reduces the distribution into liver in the IV group. Saturation is less impactful in SC doses where systemic absorption from the injection site is gradual, resulting in relatively low plasma concentrations that do not saturate ASGPR uptake. In order to compute the true absolute bioavailability, the potential for ASGPR saturation can be reduced by incorporating 1-hour infusions in place of IV bolus dosing to generate conditions similar to those observed following SC doses at pharmacologically relevant doses (McDougall et al., 2019). Optimal dose levels and sampling timepoints for an IV infusion were identified using plasma PK modeling, striking a balance between replicating the SC clearance conditions with a systemic dose while generating plasma PK profiles that can 


\section{DMD-MR-2021-000428}

accurately characterize the AUC. The systemic bioavailability of SC GaINAc-conjugated siRNA determined under this new paradigm, while variable, is much higher (on average near $100 \%$ ) than when IV bolus dosing is used as the reference and importantly is better aligned with estimated liver levels following SC dosing (Table 2). The variability in the measured absolute bioavailability underscores the complexity of attempting to use plasma PK to describe absolute systemic bioavailability particularly in the presence of active liver uptake.

\section{Biodistribution}

Given that GalNAc-siRNA is designed to target liver derived diseases and thus liver is the target organ, nonclinical studies have aimed to thoroughly characterize the pharmacokinetic parameters in liver. This has been accomplished through liver sampling during rat and monkey PKPD studies for a large number of GalNAcconjugated siRNAs. Upon absorption into systemic circulation following a SC administration, GalNAc-conjugated siRNAs are quickly and predominantly taken up by liver with liver $\mathrm{C}_{\max }$ observed around 4-8 hours in rats and 8-24 hours in monkeys. There are no significant sex-dependent differences in exposure, and the liver PK is reproducible across conjugates and linear with dose level up to $10 \mathrm{mg} / \mathrm{kg}$ (Figure $3 \mathrm{~A}$ 3J). The liver exposure significantly exceeds the plasma exposure in all cases, despite some variability in absolute amounts which suggests that there may be some sequence dependency on either the extent of liver uptake or intracellular metabolic stability. The overall liver to plasma ratio, calculated by division of measured AUC values, is high, with average ratios of 6,300 in monkey and 5,500 in rat (Table 3). In general, following SC administration of pharmacological dose levels up to $63 \%$ of the dose is recovered in 


\section{DMD-MR-2021-000428}

liver of rats and up to $87 \%$ of the dose is recovered in the liver of monkeys. The percent of dose recovered in liver decreases with increase in SC dose level. The percent of total dose in liver is also lower following IV dosing at the same dose level used in SC dosing, up to $17 \%$ of dose in rat and monkeys.

The liver half-life can be used, along with RISC loading data, to describe the pharmacodynamics of GalNAc-conjugated siRNA [

Characterizing the key PK/PD relationship in target tissue (Liver)

] and is therefore an important parameter to characterize during nonclinical studies. The liver half-lives observed for first generation conjugates (STC) ranged between 4 and $10 \mathrm{~h}$ in rats and monkeys, respectively (example using the same sequence revusiran (STC) and vutrisiran (advance ESC) is provided in Figure 10B). Liver half-lives observed with ESC, advanced ESC and ESC+ tend to overlap and range between 50$230 \mathrm{~h}$ in rats and $146-840 \mathrm{~h}$ in monkeys. The longer liver half-life in monkeys compared with rodents can be recovered by scaling $\mathrm{CL}$ with bodyweight and an exponent typically less than 1. The liver half-lives for a number of ESC molecules have also been evaluated in mice and were determined to be consistent with those derived from rats (between 37.0 and 175h).

In order to evaluate renal distribution of GalNAC-conjugated siRNAs, several studies also characterized the PK for GalNAc-siRNA in kidney. Studies in rats with siRNA2 or siRNA4 were designed to compare liver and kidney concentrations over a wide dose range. These studies showed that at pharmacologically relevant dose levels, there is very high distribution to the liver with a smaller fraction (up to 25 -fold lower) distributed to the kidney. In rat, siRNA2 liver to kidney ratios were $25,14,4.5,5.0,1.5$ and 1.5 at 1 , 


\section{DMD-MR-2021-000428}

$5,10,30,100$ and $300 \mathrm{mg} / \mathrm{kg}$, respectively. A similar observation was made for siRNA4 where liver to kidney ratios were $8.2,7.8,3.1,3.3,1.3$ and 0.85 following the same SC dose levels as siRNA2. The overall distribution between liver and kidney was dose dependent with the ratio decreasing at supratherapeutic doses; likely due to ASGPR saturation. Similar studies in monkey were consistent with studies in rat where the liver/kidney ratio decreased with increasing dose level, for example, following SC dosing of siRNA8 the ratio dropped from 57 at $30 \mathrm{mg} / \mathrm{kg}$ to 24 at $150 \mathrm{mg} / \mathrm{kg}$. Similar observations were made for two other GalNAc-siRNA, where terminal kidney levels were measured at multiple dose levels, furthermore these studies demonstrate that the ratio between liver/kidney in monkey is higher than that observed in rat.

A series of studies were conducted to characterize the mass balance, tissue distribution, excretory and metabolite profiles in rat using radiolabeled GalNAcconjugated siRNA. Due to the large molecular weight, and in order to balance the radioactive signal with the pharmacological dose level, tritium was selected as the radiolabel to support these nonclinical investigations. In the three GalNAc-conjugated siRNAs evaluated, the radiolabel was placed on an adenosine nucleotide centrally located in the antisense strand. Total radioactivity recovered in urine and feces during the studies was between 75 and 90\%. siRNA20 is an STC molecule and thus has lower stability than siRNA2 and siRNA6 which are ESC and Advanced ESC molecules, respectively. The QWBA data demonstrate that the overall distribution profiles between the three GalNAc-conjugated siRNAs evaluated are consistent with primary distribution to liver followed by much lower distribution to kidney and extra-hepatic tissues (Figure 4). There was less than $10 \%$ of the total dose recovered in the injection site by $24 \mathrm{~h}$ 


\section{DMD-MR-2021-000428}

which was consistent across studies and consistent with a study in rat using nonradiolabeled siRNA2, where $6 \%$ of the dose was recovered in tissue removed from the injection site area at 6 and $24 \mathrm{~h}$ post SC dosing of $5 \mathrm{mg} / \mathrm{kg}$ per rat.

Limited microautoradiography (MARG) data in the liver demonstrates that following a 3 $\mathrm{mg} / \mathrm{kg}$ dose the majority of radioactivity is located in Zone 1 (periportal), with moderate levels present in Zones 2 (intermediary) and 3 (centrilobular). There is also radioactivity observed at the canalicular membrane and in the bile suggestive of exocytosis into bile (Figure 4B-C). Both of these observations are consistent with findings from histopathology examinations in liver samples from rats and monkeys dosed with increasing dose levels of GalNAc-conjugated siRNA (data not shown).

The blood cell partitioning was determined during the radiolabeled excretion studies and was $\leq 1$ for the three molecules tested, indicating that GalNAc-siRNA do not preferentially distribute into blood cells.

\section{Metabolism}

To investigate the metabolic stability of the GaINAc ligand in more detail, a series of in vitro and in vivo studies were conducted. An in vitro incubation with hepatocytes from rat, monkey and human indicated faster cleavage and amidase activity in rat, while the rates observed between monkey and human were similar (Table 4). Incubations of 160 $\mu \mathrm{g} / \mathrm{mL}$ siRNA2, with $\beta$-N-acetylglucosaminidase, showed near complete loss of GalNAcmoieties by $2 \mathrm{~h}$ ( $9 \%$ remaining), whereas minimal loss was observed in serum incubations from rat, monkey and human, up to $24 \mathrm{~h}(<25 \%)$. Further in vivo plasma data shows minimal loss of GalNAc following SC administration (91\% of intact sense strand for siRNA17, $97 \%$ of intact sense strand for siRNA16). In contrast, studies 


\section{DMD-MR-2021-000428}

evaluating the $\mathrm{pH}$ dependence of GalNAc-cleavage demonstrate that higher GalNAc loss is observed under acidic $\mathrm{pH}$ conditions, $95 \%$ of intact GalNAc remained following $4 \mathrm{~h}$ incubation with mouse liver lysosomal lysate at $\mathrm{pH} 7$ versus $32 \%$ at $\mathrm{pH} 5$. Metabolite profiling of siRNA6 in liver samples from rat and monkey indicate that the sense strand is metabolized by sequential loss of GalNAc-moieties followed by cleavage of amide bonds at positions 1 (linker 1 ) thru 3 (linker 3 ), refer to Figure $1 \mathrm{~A}$. In rat, $79 \%$ of the sense strand of siRNA6 in hepatocytes lost all three GalNAc moieties at the first timepoint ( $<15$ minutes after dosing) and, importantly, no sense strand with all three GalNAc moieties was observed. After seven hours, $57 \%$ of the sense strand shows varying degrees of sequential linker metabolism, (Supplemental Figure 2). In monkey liver, 87\% of the sense strand of siRNA6 was recovered lacking all three GalNAc moieties at 8 hours post-dose. Similar to the in vitro incubation, the loss of amide bonds occurred more slowly in monkey than in rat (Supplemental Figure 2). Taken together these results demonstrate that upon SC administration of GalNAc-conjugated siRNAs, the GalNAc-moieties are quickly cleaved by $\beta$-N-acetylglucosaminidase during endo/lysosomal trafficking and the linker structure is further metabolized by amidases in hepatocytes.

Duplex siRNA undergoes metabolism through exo- and endonucleases ubiquitously distributed in plasma and tissues rather than through CYP450s (Ramsden et al., 2019). Exonucleases work on the end of the strand resulting in release of mononucleotides while endonucleases cleave internally and result in strands of varying lengths (Figure 1B). The metabolic scheme is presented in Figure 1. Metabolite profiling of plasma, urine, feces and bile from radiolabeled mass balance studies demonstrate that siRNA6 


\section{DMD-MR-2021-000428}

was cleared in rats via a combination of metabolism and renal and biliary clearance of parent drug. In plasma, unchanged siRNA6 accounted for approximately $75 \%$ of the total radioactivity exposure through 96 hours (AUC0-96) postdose. $3^{\prime} \mathrm{N}-1$ contributed $6.86 \%$ of the of the total radioactivity exposure. Radioprofiles of urine samples showed that unchanged siRNA6 accounted for 3.71 and $5.75 \%$ of the dose from intact and BDC rats, respectively. $3^{\prime} \mathrm{N}-1$ accounted for $0.213 \%$ of the dose in urine from intact rats. Approximately 22.9 and $14.0 \%$ of the dose in intact and BDC rats, respectively, eluted at the void volume. Efforts to identify the components of this peak were unsuccessful (M1), but the peak likely comprises multiple dimers or shortmers containing the radiolabel and not a single metabolite. Approximately $9 \%$ of the dose was recovered as unchanged siRNA6 in bile. $3^{\prime} \mathrm{N}-1$ accounted for approximately $3 \%$ of the dose in bile. All the radioactivity recovered in feces was associated with unknown components. No unchanged siRNA6 was recovered in feces from intact rats. [3H]-siRNA6 was metabolized in rats after subcutaneous administration via hydrolysis. Most of the $[3 \mathrm{H}]-$ siRNA20-derived radioactivity was excreted after subcutaneous administration over 168 hours after dosing (intact rats), primarily in urine. After subcutaneous administration to intact rats, means of 67 and $7 \%$ of the administered radioactivity were excreted in urine and feces, respectively, by 1344 hours postdose. In BDC rats after subcutaneous dosing, means of $57.0,16.6$, and $1.91 \%$ of the administered radioactivity were excreted in urine, bile, and feces, respectively, by 168 hours postdose. A large amount of radioactivity was eliminated in urine after subcutaneous dosing indicating that renal excretion was a major route of elimination of $[3 \mathrm{H}]$-siRNA20-derived radioactivity. The analysis of urine and bile samples for radioactivity before and after drying indicated the 


\section{DMD-MR-2021-000428}

fraction of radioactivity in the form of tritiated water generally increased over time for urine and bile. The mean overall recoveries of radioactivity after subcutaneous dosing to intact and BDC rats were 82.8 and $89.2 \%$, respectively. It is possible that the lower recovery of radioactivity was due to expiry of tritiated water. Radioprofiles of urine samples showed that unchanged siRNA20 accounted for 1.67 and $0.805 \%$ of the dose from intact and BDC rats, respectively. $3^{\prime} \mathrm{N}-1$ accounted for 0.555 and $2.84 \%$ of the dose in urine from intact and BDC rats, respectively. Approximately 56 and $46 \%$ of the dose in intact and BDC rats, respectively, eluted at the void volume. Efforts to identify the components of early eluting peaks, M1/M2, were unsuccessful, but the peaks likely comprised multiple dimers or shortmers containing the radiolabel (not a single metabolite) and also a considerable portion of tritiated water based on the urine drying experiment. In bile, approximately $1 \%$ of the dose was recovered as unchanged siRNA20. $3^{\prime} \mathrm{N}-1$ was recovered in bile and accounted for approximately $1 \%$ of the dose. $[3 \mathrm{H}]$-siRNA20 was metabolized in rats after subcutaneous administration via hydrolysis. Four metabolites were tentatively identified, in addition to $3^{\prime} \mathrm{N}-1$ and unchanged siRNA20. Metabolite profiling and identification results indicate that siRNA20 was primarily cleared in rats via metabolism. Loss of a single nucleotide from the $3^{\prime}$-end of the antisense strand, annotated as $3^{\prime}$ ' $\mathrm{N}-1(\mathrm{AS})$, is an often-observed metabolite in liver samples confirmed by mass spectrometry analysis (Figure 5). The $3^{\prime} \mathrm{N}-1$ (AS) metabolite is typically as potent as the parent molecule (Table 5). Formation of this active metabolite in monkey liver can differ across GalNAc-conjugates targeting different mRNAs (Figure 5). Additional truncation of metabolites can range in activity compared with the full-length siRNA with $5^{\prime}$ nucleotide loss generally rendering inactive 


\section{DMD-MR-2021-000428}

metabolites (Table 5). Limited data has been generated which confirms that loading efficiency of the active metabolites into RISC is equivalent to the parent compound. The formation of metabolites appears to be conserved across species enabling extrapolation between in vitro and in vivo data (Table 4, Supplemental Table 2).

\section{Excretion}

The mass balance data from radiolabeled ADME studies in rat coupled with metabolite profiling data from plasma, urine, feces and bile demonstrate that most of the radioactivity was excreted as truncated metabolites in urine, with a small fraction $(<25 \%)$ representing intact antisense strand. These results are consistent with the

urinary excretion data obtained using unlabeled GaINAc-conjugated siRNAs. In bile duct cannulated rats, the radioactivity is eliminated primarily through truncated metabolites and full length antisense to a limited extent. The radioactivity recovered in feces of intact rats was less than the amount of radioactivity excreted in bile of BDC rats suggesting that some truncated metabolites can undergo enterohepatic reabsorption. Data across five GalNAc-conjugated siRNAs including radiolabeled mass balance studies demonstrate that the extent of biliary clearance of intact siRNA is limited (10-20\%). A caveat, when interpreting these data, is that biliary excretion was only measured for one week in cannulated rats. In addition, depending on where the radiolabel is placed, there can be a classic double peak, suggestive of enterohepatic recirculation (Figure 6A). Metabolite profiling of samples taken during the double peak reveal that the majority of radioactivity is contained within shortmer metabolites and no peak corresponding to active metabolites or full-length GalNAc-conjugated siRNA2 is observed (Figure 6B). PD evaluation in bile-duct cannulated rats shows that there is no impact on PD 
DMD-MR-2021-000428

suggesting that any excreted full-length siRNA does not undergo hepatobiliary recycling to further contribute to PD (Figure 7). This is consistent with the GalNAc metabolism data demonstrating that the GalNAc moieties are quickly cleaved under acidic conditions during endocytic trafficking.

Renal excretion of full-length siRNA, as a percent of total drug administered, has been investigated for multiple GalNAc-conjugated siRNA in nonclinical models including rat and monkey during routine PK studies following SC administration (Table 6). Across different conjugates designed for various targets the urinary excretion was less than $25 \%$. This was also the case when these molecules were dosed to humans (Sagar Agarwal et al., 2020; Habtemariam et al., 2020).

To investigate whether reduced kidney function may lead to changes in plasma or liver PK, renal excretion or PD outcomes, the 5/6 nephrectomy model was evaluated. The $5 / 6$ nephrectomy model has been successfully employed to evaluate the impact of chronic renal failure on designated end-points, including toxicity and PK (Glund et al., 2018; Kujal \& Vernerová, 2008; P. Li et al., 2012; Ozcan et al., 2012; Tapia et al., 2013). This model was successfully qualified, in our laboratory, and aggregate analysis of three nephrectomy studies demonstrates no meaningful differences in the plasma and liver PK, or PD of GalNAc-conjugated siRNA in rats with and without nephrectomy. While there was a decrease in urinary excretion of full-length GalNAc-conjugated siRNA in rats with nephrectomy relative to those without nephrectomy, these data confirmed that renal excretion plays a minor role in the overall elimination of RNAi therapeutics. Importantly, moderate to severe renal impairment does not influence the liver concentration and target mRNA reduction (Table 7). 


\section{DMD-MR-2021-000428}

\section{Characterizing the key PK/PD relationship in target tissue (Liver)}

The PKPD relationship of GalNAc-conjugated siRNA is more complex than conventional small molecules as plasma PK does not correlate directly with the PD effect. First, there is a significant difference between plasma $\mathrm{PK} \mathrm{T}_{\max }$ and the corresponding $\mathrm{PD} \mathrm{T}_{\max }$. Plasma $T_{\max }$ from SC doses is achieved quickly, between $0.2-12 \mathrm{~h}$, while PD $\mathrm{T}_{\max }$ occurs later, typically at least 3 days after dosing in mice, a week after dosing in rat, and 2 weeks after dosing in monkey (Figure 9, representative data; additional examples not shown). Liver $T_{\max }$, occurs later than plasma $T_{\max }$, typically between 8-24 hours post dose in all species, but still proceeds PD $T_{\max }$ significantly. Only the kinetics of siRNA in RISC, with a $T_{\max }$ coincidental with maximal PD response, shows a direct relationship between PK $T_{\max }$ and PD $T_{\max }$ (Figure $8 C, D$ ). A second complexity results from a disconnect between the plasma PK half-life, less than 8 hours in all species (Table 1), and the prolonged duration of the PD remaining for weeks in rodents and up to several months in monkeys and humans. PD effect profiles correspond much more directly with the liver half-life and siRNA exposure in RISC than the plasma half-life (Figure 8), and the metabolic stability of siRNA in liver has been key to realizing long term stable target reduction with infrequent dosing.

When the same GalNAc-siRNA was active across multiple species, the onset of activity in rodents occurs earlier than in monkeys and humans, and the duration of effect was shorter. Similarly, the duration of pharmacological activity was shorter in monkey than in human (Figure 9). The plasma, liver and RISC PK can be scaled across species allometrically, with higher clearance rates observed in mice/rats compared to monkeys and humans. An example of this is provided for revusiran, an STC molecule, and 


\section{DMD-MR-2021-000428}

vutrisiran, an advanced ESC molecule (Habtemariam et al., 2020; Zimmermann et al., 2017), (Figure 10). Both of these are GalNAc-conjugated siRNAs and contain the same sequence targeting human transthyretin mRNA (hTTR). In humans, revusiran required a 5-day daily loading dose (500 $\mathrm{mg}$ ) followed by weekly dosing where as vutrisiran can be dosed in humans at $25 \mathrm{mg}$ every three months and maintain similar reduction of circulating TTR levels throughout. As depicted in Figure 10, the impact of improved metabolic stability of vutrisiran results in increased liver exposure (Figure 10A), and half-life (Figure 10B) in monkey, which translates to durable pharmacodynamics following reduced dose levels and frequency (Figures 10C). This is reproduced during human clinical trials where, once dosing of revusiran is stopped, the TTR levels swiftly recovered whereas the TTR reductions were maintained for several months following single doses of vutrisiran (Figure 10D) (Habtemariam et al., 2020). In vitro data, generated in serum and S9 from rat, monkey and human, also showed a trend for lower metabolic stability with revusiran compared with vutrisiran although was not quantitative with respect to predicting half-life.

The direct PD driver of GalNAc-conjugated siRNA drugs is the amount of siRNA (antisense strand) loaded in RISC, with the observed PD effect (as measured by reduction of target protein in plasma) corresponding directly with the concentration of RISC-loaded siRNA (Figures $8 \mathrm{C}$ and $8 \mathrm{D}$ ). RISC $\mathrm{T}_{\max }$ typically ranges between 3-7 days in both rats and monkeys. The RISC $\mathrm{C}_{\max }$ is significantly lower than that in liver, often by 1000-fold or more (Castellanos-Rizaldos et al., 2020). 


\section{DMD-MR-2021-000428}

\section{Discussion:}

For the development of RNAi therapeutics, understanding the translation of nonclinical PK/PD and ADME properties across compounds and species enables prediction and subsequent understanding of these attributes in humans. The majority of the siRNAs currently in clinical development are conjugated to GaINAc ligands for targeted delivery to the liver. Here we describe the development of the tools and the design of studies to (i) investigate the similarity of absorption, biodistribution, metabolism, and excretion properties across compounds and species; (ii) characterize metabolic stability and its impact on dosing regimen; and (iii) elucidate siRNA exposures in liver and RISC as direct drivers of PD which are used to project human efficacious dose through allometric scaling. These nonclinical evaluations have assisted the optimization of chemical modifications and delivery systems ultimately resulting in the approval of four RNAi therapeutics and proof of clinical efficacy for many more currently in late stage clinical development (Debacker et al., 2020).

Several recent publications have highlighted the bioanalytical approaches used to quantify levels of GalNAc-conjugated siRNA from various biological matrices (Castellanos-Rizaldos et al., 2020; J. Li et al., 2019; Liu et al., 2019). Given the instability observed for single strands under in vitro assay conditions and in vivo matrices employed during nonclinical evaluations, measuring the antisense strand alone can be taken as reflective of the duplex, eliminating the need to characterize both the sense and antisense strand and improving throughput.

In order to characterize the ADME properties for GalNAc-conjugated siRNA, many nonclinical studies in rats and monkeys have been conducted across current chemistry 


\section{DMD-MR-2021-000428}

platforms (ESC, Advanced ESC and ESC+) on more than 22 GalNAc-conjugated siRNAs containing various sequences and targeting different proteins. The aggregate analysis of these results demonstrates that, at clinically relevant dose levels, there are no sex dependent differences in plasma or liver PK parameters and exposures increase in a dose proportional manner. Furthermore, across various conjugates, the overall dose-normalized exposures are primarily within two-fold of the mean for plasma, liver and RISC in rat and monkeys resulting in highly predictable and extrapolatable plasma and liver PK properties. This enables prediction of expected plasma, liver and RISC loaded concentrations for different siRNA molecules while designing toxicology and first-in-human studies. Additionally, the plasma exposure in humans can be predicted from data generated in rats and monkeys providing confidence in predictions made for human liver PK through allometric scaling from nonclinical models (manuscript in process).

The assessment of absolute systemic bioavailability is complicated by the rapid distribution into liver, resulting in low apparent plasma concentrations following SC doses which are not reflective of overall absorption coupled with saturation of liver uptake with IV bolus dosing. Therefore, traditional bioavailability studies relying on IV bolus administration at relatively high doses underestimate the bioavailability of SC administered GalNAc-conjugated siRNA. When evaluating the liver levels at $\mathrm{C}_{\max }$ as a percent of the total dose it becomes clear that much greater levels of GalNAcconjugated siRNA are absorbed following SC administration than the measured bioavailability values would suggest. In order to derive a true bioavailability estimate, studies were designed with optimized infusion time to limit ASGPR saturation while still 
DMD-MR-2021-000428

enabling adequate plasma exposure to characterize the AUC and multiple recent studies, conducted with this new paradigm, suggest near complete bioavailability of GalNAc-conjugated siRNA following SC administration. The large variability in these infusion experiments, however, suggests plasma PK based method is not a reliable approach to assess the true bioavailability of GalNAc-conjugated siRNA.

Radiolabeled ADME studies conducted in rats have served as a surrogate to conducting these studies in humans. This is primarily driven by the knowledge gained from studies with cold (unlabeled) compounds, which demonstrate cross-species translatability, and the prolonged radioactive exposure in liver that makes human mass balance studies unethical. The distribution pattern for GalNAc conjugates in QWBA studies shows that the majority of radiolabeled GalNAc-conjugated siRNA is observed in the liver, with lower levels in extrahepatic tissues including kidney, lymph nodes, adrenal gland, pancreas, jejunum and bone marrow. There was no radioactivity observed in the brain and negligible levels in the heart, largely since ASGPR expression has been shown to be primarily enriched in the liver (Carito et al., 2016). Data presented in Brown et al., 2020, demonstrates that GalNAc-conjugated siRNAs localize in acidic vesicular compartments, likely late endosomes and lysosomes. Radiolabeled ADME studies coupled with MARG data reveal dose dependency in the pattern of distribution within hepatocytes with higher dose levels achieving more uniform distribution across zones whereas with lower dose levels (i.e. $3 \mathrm{mg} / \mathrm{kg}$ ), higher distribution is observed in Zone 1 and moderate levels in Zones 2 and 3. MARG analysis of siRNA6 also confirmed localization at the bile duct which is consistent with release into bile through exocytosis of lysosomal content. This result is also consistent with histopathology examinations 
DMD-MR-2021-000428

from rat and monkey liver samples. Negligible levels are observed in cardiac tissue, which taken together with the large molecular size, lack of hERG channel blockade and no evidence of QT prolongation in clinical studies obviate the need to conduct dedicated QT studies in the clinic. Furthermore, upon SC administration limited amounts $(<10 \%)$ are retained at the injection site or in the lymphatic system limiting the potential for delayed high concentrations released into systemic circulation.

Plasma protein binding has been extensively covered in prior publications using an EMSA method (Rocca et al., 2019). The data demonstrate that PPB appears to be $>85 \%$ at clinically relevant concentration levels but is conserved across species, does not limit active ASGPR-mediated uptake (Saket Agarwal et al., 2021) and is saturable at toxicological dose levels (>100 mg/kg) (Rocca et al., 2019). This results in higher "free" GalNAc-conjugated siRNA in the GLP toxicology studies (fraction unbound $~ 50 \%$ ), than is observed in the clinical dose range (1-20\% fraction unbound). Therefore, safety margins in nonclinical species based on total siRNA concentrations likely include a conservative estimate. Furthermore, studies using protein pull down approaches have determined that GalNAc-siRNA does not bind to albumin but does bind to multiple other proteins present in plasma (Saket Agarwal et al., 2021).

Nonclinical studies in ASGR1 knockout mice confirm that functional ASGPR is required for optimal PD activity of GalNAc-conjugated siRNAs, and in the absence of productive liver uptake both plasma and kidney exposure increase significantly as does urinary excretion. Similar observations were made in wild-type mice dosed with the same siRNA containing linker but not GalNAc (Liu et al., submitted). Previous studies have demonstrated that despite 50\% reduction in ASGPR levels, pharmacodynamic activity is 


\section{DMD-MR-2021-000428}

maintained suggesting that receptor capacity is sufficient to efficiently internalize GalNAc-conjugated siRNA (Willoughby et al., 2018). Although there are literature reports demonstrating relevant changes in hepatic expression of ASGPR in various disease states (Matsuzaki et al., 1997; Reimer et al., 1991; Witzigmann et al., 2016), the data generated to date for liver directed siRNA supports a tiered approach to evaluate the potential impact of hepatic impairment, whereby data is first generated in patients with mild hepatic impairment prior to evaluation of PD, safety and efficacy in moderate and severe hepatic impairment.

The metabolite profile, while slightly different across conjugates, does appear to be well conserved across species with higher nuclease activity observed in rodents than in monkey and human. In plasma, the current chemical modifications result in limited metabolism overall $(<10 \%)$ and are similar across conjugates and species. In general, metabolites formed in the liver are expected to contribute to the overall efficacy and are therefore considered in extrapolation of PD from PK.

Excretion data from both radiolabeled and cold studies in rats and monkeys confirm that the majority of the GalNAc-conjugated siRNA is metabolized slowly in liver and excreted as shortmer metabolites through urine and bile. Full length and truncated metabolites excreted into bile undergo further degradation by endo and exo-nucleases in the gastrointestinal (GI) tract and shortmer metabolites can be reabsorbed into systemic circulation. An important consideration during clinical development is related to whether renal impairment may impact the pharmacokinetics of the drug, which could lead to higher systemic exposure but no impact on liver uptake or subsequent PD (Wright et al., 2020). Studies in the $5 / 6$ nephrectomy rat model, recapitulating moderate to severe 


\section{DMD-MR-2021-000428}

renal impairment, demonstrate that a reduction in urinary excretion does not lead to marked changes in liver PK or observed PD although transient increases in plasma exposure may be observed. Therefore, renal impairment is unlikely to influence the liver PK and subsequent PD of GalNAc-conjugated siRNAs and clinical evaluation is often not warranted (Wright et al., 2020).

The PK/PD relationship of GalNAc-conjugated siRNA is more complex than conventional small molecule drugs. This is exemplified by the delayed onset of action in contrast to the rapid absorption and short plasma half-life observed in nonclinical models and human. Significant understanding has been gained in describing the relationship between dose and exposure in plasma, liver and RISC using nonclinical models. It is now recognized that slow release of metabolically stable siRNAs and active metabolites into the cytoplasm from the acidic compartments is key to describing the duration of the PD response. With the success of GalNAc-conjugated siRNAs in treating diseases in the clinic, it's apparent that many of the key ADME properties learned from nonclinical investigations can be translated to predict ADME, liver and RISC PK in humans.

In summary, the ADME and PK/PD properties of several GalNAc-conjugated siRNAs have been extensively characterized in vitro and in vivo. These data demonstrate that GalNAc-conjugated siRNAs possess ADME and PK/PD properties that are generally well conserved across species, which form the foundation for translation of clinical dose and dosing regimen in the absence of PK and PD data directly derived from human liver. SC administration of GalNAc-conjugated siRNAs results in targeted delivery to the 


\section{DMD-MR-2021-000428}

intended organ (liver) with a long-lasting PK/PD duration suitable for infrequent dosing in the clinic for this new class of therapeutic modalities. 


\section{DMD-MR-2021-000428}

\section{Acknowledgements}

The authors thank the Alnylam In Vivo Sciences and Bioanalytical groups and acknowledge current and former members of the Alnylam Early Development department, the Platform Core Team and the support of senior management.

\section{Authorship Contributions}

Participated in research design: McDougall, Ramsden, Agarwal, Agarwal, Brown, Castellanos-Rizaldos, Charisse, Chong, Cichocki, Fitzgerald, Goel, Guenther, Habtemariam, Jadhav, Janas, Jayaraman, Kurz, Li, Liu J, Maclauchlin, Maier, Manoharan, Nair, Robbie, Smith, Theile, Vaishnaw, Xu, Zlatev and Wu.

Conducted experiments: Ramsden, Agarwal, Aluri, Arciprete, Brown, Gu, Li, Liu J, Liu X, Liou, Manoharan, Schmidt, Theile, Waldron, Zhang

Performed data analysis: McDougall, Ramsden, Agarwal, Brown, Castellanos-Rizaldos, Chong, Cichocki, Zhang

Wrote or contributed to the writing of the manuscript: McDougall, Ramsden, Chong, Habtemariam, Jadhav, Fitzgerald, Maier, Robbie, Zlatev, Wu 


\section{DMD-MR-2021-000428}

\section{References:}

Agarwal, Sagar, Simon, A. R., Goel, V., Habtemariam, B. A., Clausen, V. A., Kim, J. B., \& Robbie, G. J. (2020). Pharmacokinetics and Pharmacodynamics of the Small Interfering Ribonucleic Acid, Givosiran, in Patients With Acute Hepatic Porphyria. Clinical Pharmacology and Therapeutics, 108(1), 63-72. https://doi.org/10.1002/cpt.1802

Agarwal, Saket, Allard, R., Darcy, J., Chigas, S., Gu, Y., Nguyen, T., Bond, S., Chong, S., Wu, J.-T., \& Janas, M. M. (2021). Impact of Serum Proteins on the Uptake and RNA Interference Activity of $\mathrm{N}$-Acetylgalactosamine-Conjugated Small Interfering RNAs. Nucleic Acid Therapeutics. https://doi.org/10.1089/nat.2020.0919

Brown, C. R., Gupta, S., Qin, J., Racie, T., He, G., Lentini, S., Malone, R., Yu, M., Matsuda, S., Shulga-Morskaya, S., Nair, A. V, Theile, C. S., Schmidt, K., Shahraz, A., Goel, V., Parmar, R. G., Zlatev, I., Schlegel, M. K., Nair, J. K., ... Jadhav, V. (2020). Investigating the pharmacodynamic durability of GalNAc-siRNA conjugates. Nucleic Acids Research, 48(21), 11827-11844. https://doi.org/10.1093/nar/gkaa670

Carito, B., Harbison, C., Janas, M., \& Keirstead, N. (2016). Targeted Delivery of siRNA Therapeutics: ASGPR Tissue Expression Evaluation in Rat, Mouse, and Non-human Primate. ACVP \& ASVCP Concurrent Annual Meeting 2016 (Poster).

Castellanos-Rizaldos, E., Brown, C. R., Dennin, S., Kim, J., Gupta, S., Najarian, D., Gu, Y., Aluri, K., Enders, J., Brown, K., \& Xu, Y. (2020). RT-qPCR Methods to Support Pharmacokinetics and Drug Mechanism of Action to Advance Development of RNAi Therapeutics. Nucleic Acid Therapeutics, 30(3), 133-142.

https://doi.org/10.1089/nat.2019.0840

Chen, C., Ridzon, D. A., Broomer, A. J., Zhou, Z., Lee, D. H., Nguyen, J. T., Barbisin, M., Xu, N. L., Mahuvakar, V. R., Andersen, M. R., Lao, K. Q., Livak, K. J., \& Guegler, K. J. (2005). Real-time quantification of microRNAs by stem-loop RT-PCR. Nucleic Acids Research, 33(20), e179. https://doi.org/10.1093/nar/gni178 


\section{DMD-MR-2021-000428}

Debacker, A. J., Voutila, J., Catley, M., Blakey, D., \& Habib, N. (2020). Delivery of Oligonucleotides to the Liver with GalNAc: From Research to Registered Therapeutic Drug. Molecular Therapy, 28(8), 1759-1771. https://doi.org/10.1016/j.ymthe.2020.06.015

Fire, A., Xu, S., Montgomery, M. K., Kostas, S. A., Driver, S. E., \& Mello, C. C. (1998). Potent and specific genetic interference by double-stranded RNA in Caenorhabditis elegans. Nature, 391(February), 806-811. https://www.nature.com/articles/35888.pdf

Foster, D. J., Brown, C. R., Shaikh, S., Trapp, C., Schlegel, M. K., Qian, K., Sehgal, A., Rajeev, K. G., Jadhav, V., Manoharan, M., Kuchimanchi, S., Maier, M. A., \& Milstein, S. (2018). Advanced siRNA Designs Further Improve In Vivo Performance of GaINAc-siRNA Conjugates. Molecular Therapy, 26(3), 708-717. https://doi.org/10.1016/j.ymthe.2017.12.021

Glund, S., Gan, G., Moschetti, V., Reilly, P., Honickel, M., Grottke, O., \& Van Ryn, J. (2018). The Renal Elimination Pathways of the Dabigatran Reversal Agent Idarucizumab and its Impact on Dabigatran Elimination. Clinical and Applied Thrombosis/Hemostasis, 24(5), 724-733. https://doi.org/10.1177/1076029618755947

Habtemariam, B. A., Karsten, V., Attarwala, H., Goel, V., Melch, M., Clausen, V. A., Garg, P., Vaishnaw, A. K., Sweetser, M. T., Robbie, G. J., \& Vest, J. (2020). Single-Dose Pharmacokinetics and Pharmacodynamics of Transthyretin Targeting Nacetylgalactosamine-Small Interfering Ribonucleic Acid Conjugate, Vutrisiran, in Healthy Subjects. Clinical Pharmacology and Therapeutics, 109(2), 372-382.

https://doi.org/10.1002/cpt.1974

Hail, M. E., Eliiot, B., \& Anderson, K. (2004). High-Throughput Analysis of Oligonucleotides Using Automated Electrospray Ionization Mass Spectrometry. American Biotechnology Laboratory, 22(1), 12-14. https://www.enovatia.com/wpcontent/uploads/ABL_OligoHTCS_article.pdf Janas, M. M., Schlegel, M. K., Harbison, C. E., Yilmaz, V. O., Jiang, Y., Parmar, R., Zlatev, I., 


\section{DMD-MR-2021-000428}

Castoreno, A., Xu, H., Shulga-Morskaya, S., Rajeev, K. G., Manoharan, M., Keirstead, N. D., Maier, M. A., \& Jadhav, V. (2018). Selection of GalNAc-conjugated siRNAs with limited off-Target-driven rat hepatotoxicity. Nature Communications, 9(1), 723. https://doi.org/10.1038/s41467-018-02989-4

Kliem, V., Johnson, R. J., Alpers, C. E., Yoshimura, A., Couser, W. G., Koch, K. M., \& Floege, J. (1996). Mechanisms involved in the pathogenesis of tubulointerstitial fibrosis in 5/6nephrectomized rats. Kidney International, 49(3), 666-678.

https://doi.org/10.1038/ki.1996.95

Kujal, P., \& Vernerová, Z. (2008). 5/6 Nephrectomy as an experimental model of chronic renal failure and adaptation to reduced nephron number. Ceskoslovenská Fysiologie / Ústrední Ústav Biologický, 57, 104-109.

Landesman, Y., Svrzikapa, N., Cognetta, A., Zhang, X., Bettencourt, B. R., Kuchimanchi, S., Dufault, K., Shaikh, S., Gioia, M., Akinc, A., Hutabarat, R., \& Meyers, R. (2010). In vivo quantification of formulated and chemically modified small interfering RNA by heating-inTriton quantitative reverse transcription polymerase chain reaction (HIT qRT-PCR). Silence, 1(1), 1-14. https://doi.org/10.1186/1758-907X-1-16

Li, J., Liu, J., Enders, J., Arciprete, M., Tran, C., Aluri, K., Guan, L. H., O’Shea, J., Bisbe, A., Charissé, K., Zlatev, I., Najarian, D., \& Xu, Y. (2019). Discovery of a novel deaminated metabolite of a single-stranded oligonucleotide in vivo by mass spectrometry. Bioanalysis, 11(21), 1955-1965. https://doi.org/10.4155/bio-2019-0118 Li, P., Ma, L. L., Xie, R. J., Xie, Y. S., Wei, R. B., Yin, M., Wang, J. Z., \& Chen, X. M. (2012). Treatment of 5/6 nephrectomy rats with sulodexide: A novel therapy for chronic renal failure. Acta Pharmacologica Sinica, 33(5), 644-651. https://doi.org/10.1038/aps.2012.2 Liu, J., Li, J., Tran, C., Aluri, K., Zhang, X., Clausen, V., Zlatev, I., Guan, L., Chong, S., Charisse, K., Wu, J. T., Najarian, D., \& Xu, Y. (2019). Oligonucleotide quantification and metabolite profiling by high-resolution and accurate mass spectrometry. Bioanalysis, 


\section{DMD-MR-2021-000428}

11(21), 1967-1980. https://doi.org/10.4155/bio-2019-0137

Matsuzaki, S., Onda, M., Tajiri, T., \& Kim, D. Y. (1997). Hepatic lobar differences in progression of chronic liver disease: Correlation of asialoglycoprotein scintigraphy and hepatic functional reserve. Hepatology, 25(4), 828-832. https://doi.org/10.1002/hep.510250407

McDougall, R., Ramsden, D., Chong, S., \& Wu, J.-T. (2019). ADME and PK Properties of GalNAc-conjugated siRNAs in Nonclinical Species. In Presented at High-Throughput ADME Conference (June), Cambridge MA.

Meister, G., \& Tuschl, T. (2004). Mechanisms of gene silencing by double-stranded RNA. Nature, 431(7006), 343-349. https://doi.org/10.1038/nature02873

Nair, J. K., Attarwala, H., Sehgal, A., Wang, Q., Aluri, K., Zhang, X., Gao, M., Liu, J., Indrakanti, R., Schofield, S., Kretschmer, P., Brown, C. R., Gupta, S., Willoughby, J. L. S., Boshar, J. A., Jadhav, V., Charisse, K., Zimmermann, T., Fitzgerald, K., ... Maier, M. A. (2017). Impact of enhanced metabolic stability on pharmacokinetics and pharmacodynamics of GalNAc-siRNA conjugates. Nucleic Acids Research, 45(19), 10969-10977. https://doi.org/10.1093/nar/gkx818

Nair, J. K., Willoughby, J. L. S., Chan, A., Charisse, K., Alam, M. R., Wang, Q., Hoekstra, M., Kandasamy, P., Kelin, A. V., Milstein, S., Taneja, N., Oshea, J., Shaikh, S., Zhang, L., Van Der Sluis, R. J., Jung, M. E., Akinc, A., Hutabarat, R., Kuchimanchi, S., .. Manoharan, M. (2014). Multivalent N -acetylgalactosamine-conjugated siRNA localizes in hepatocytes and elicits robust RNAi-mediated gene silencing. Journal of the American Chemical Society, 136(49), 16958-16961. https://doi.org/10.1021/ja505986a

Ozcan, A., Ware, K., Calomeni, E., Nadasdy, T., Forbes, R., Satoskar, A. A., Nadasdy, G., Rovin, B. H., Hebert, L. A., \& Brodsky, S. V. (2012). 5/6 Nephrectomy As a Validated Rat Model Mimicking Human Warfarin-Related Nephropathy. American Journal of Nephrology, 35(4), 356-364. https://doi.org/10.1159/000337918

Ramsden, D., Wu, J. T., Zerler, B., Iqbal, S., Jiang, J., Clausen, V., Aluri, K., Gu, Y., Dennin, S., 


\section{DMD-MR-2021-000428}

Kim, J., \& Chong, S. (2019). In vitro drug-drug interaction evaluation of GalNAc conjugated siRNAs against CYP450 enzymes and transporters. Drug Metabolism and Disposition, 47(10), 1183-1194. https://doi.org/10.1124/dmd.119.087098

Ray, K. K., Wright, R. S., Kallend, D., Koenig, W., Leiter, L. A., Raal, F. J., Bisch, J. A., Richardson, T., Jaros, M., Wijngaard, P. L. J., \& Kastelein, J. J. P. (2020). Two Phase 3 Trials of Inclisiran in Patients with Elevated LDL Cholesterol. New England Journal of Medicine, 382(16), 1507-1519. https://doi.org/10.1056/nejmoa1912387

Reimer, P., Weissleder, R., Lee, A. S., Buettner, S., Wittenberg, J., \& Brady, T. J. (1991). Asialoglycoprotein receptor function in benign liver disease: evaluation with MR imaging. Radiology, 178(3), 769-774. https://doi.org/10.1148/radiology.178.3.1994416

Rocca, C., Dennin, S., Gu, Y., Kim, J., Chigas, S., Najarian, D., Chong, S., Gutierrez, S., Butler, J., Charisse, K., Robbie, G., Xu, Y., \& Brown, K. (2019). Evaluation of electrophoretic mobility shift assay as a method to determine plasma protein binding of siRNA. Bioanalysis, 11(21), 1927-1939. https://doi.org/10.4155/bio-2019-0151

Rudge, J., Scott, G., Hail, M., \& Mcginley, M. (2011). Preparation and LC / MS Analysis of Oligonucleotide Therapeutics from Biological Matrices. Chromatography Today, March, 16-20.

Schlegel, M. K., Foster, D. J., Kel'In, A. V., Zlatev, I., Bisbe, A., Jayaraman, M., Lackey, J. G., Rajeev, K. G., Charissé, K., Harp, J., Pallan, P. S., Maier, M. A., Egli, M., \& Manoharan, M. (2017). Chirality Dependent Potency Enhancement and Structural Impact of Glycol Nucleic Acid Modification on siRNA. Journal of the American Chemical Society, 139(25), 85378546. https://doi.org/10.1021/jacs.7b02694

Shen, X., \& Corey, D. R. (2018). Chemistry, mechanism and clinical status of antisense oligonucleotides and duplex RNAs. Nucleic Acids Research, 46(4), 1584-1600. https://doi.org/10.1093/nar/gkx1239

Tapia, E., Zatarain-Barrón, Z. L., Hernández-Pando, R., Zarco-Márquez, G., Molina-Jijón, E., 


\section{DMD-MR-2021-000428}

Cristóbal-García, M., Santamaría, J., \& Pedraza-Chaverri, J. (2013). Curcumin reverses glomerular hemodynamic alterations and oxidant stress in 5/6 nephrectomized rats. Phytomedicine, 20(3-4), 359-366. https://doi.org/10.1016/j.phymed.2012.11.014

Willoughby, J. L. S., Chan, A., Sehgal, A., Butler, J. S., Nair, J. K., Racie, T., Shulga-Morskaya, S., Nguyen, T., Qian, K., Yucius, K., Charisse, K., van Berkel, T. J. C., Manoharan, M., Rajeev, K. G., Maier, M. A., Jadhav, V., \& Zimmermann, T. S. (2018). Evaluation of GalNAc-siRNA Conjugate Activity in Pre-clinical Animal Models with Reduced Asialoglycoprotein Receptor Expression. Molecular Therapy, 26(1), 105-114. https://doi.org/10.1016/j.ymthe.2017.08.019

Witzigmann, D., Quagliata, L., Schenk, S. H., Quintavalle, C., Terracciano, L. M., \& Huwyler, J. (2016). Variable asialoglycoprotein receptor 1 expression in liver disease: Implications for therapeutic intervention. Hepatology Research, 46(7), 686-696.

https://doi.org/https://doi.org/10.1111/hepr.12599

Wright, R. S., Collins, M. G., Stoekenbroek, R. M., Robson, R., Wijngaard, P. L. J., Landmesser, U., Leiter, L. A., Kastelein, J. J. P., Ray, K. K., \& Kallend, D. (2020). Effects of Renal Impairment on the Pharmacokinetics, Efficacy, and Safety of Inclisiran: An Analysis of the ORION-7 and ORION-1 Studies. Mayo Clinic Proceedings, 95(1), 77-89. https://doi.org/10.1016/j.mayocp.2019.08.021

Zimmermann, T. S., Karsten, V., Chan, A., Chiesa, J., Boyce, M., Bettencourt, B. R., Hutabarat, R., Nochur, S., Vaishnaw, A., \& Gollob, J. (2017). Clinical Proof of Concept for a Novel Hepatocyte-Targeting GalNAc-siRNA Conjugate. Molecular Therapy, 25(1), 71-78. https://doi.org/10.1016/j.ymthe.2016.10.019 


\section{DMD-MR-2021-000428}

\section{Footnotes}

- This work was supported by Alnylam Pharmaceuticals

- The authors have no conflict of interest but are current or former employees of Alnylam Pharmaceuticals 


\section{DMD-MR-2021-000428}

\section{FIGURE LEGENDS}

Visual Abstract: The overall absorption, distribution, metabolism and excretion profile for GalNAc-conjugated siRNA has been elucidated through the conduct of nonclinical in vitro and in vivo investigations. Following SC administration, at pharmacological dose levels, minimal GalNAc-conjugated siRNA is retained at the injection site $(<10 \%)$ with majority reaching systemic circulation where it is rapidly taken up by liver, the target organ. GalNAc-conjugated siRNA not taken up into the liver is excreted intact into urine $(<25 \%)$ or minimally distributed across other tissues. Once taken up into clathrin-coated vesicles by the high capacity ASGPR, which is predominately expressed on the cell surface of hepatocytes, the GalNAc-conjugated siRNA is trafficked into endosomal compartments where it can be further metabolized via ligand deglycosylation and amidases, and thru exo- and endonucleases. The majority of metabolism occurs in the hepatocytes with metabolites undergoing elimination thru bile or urine. Intact duplex which is slowly released into cytoplasm is able to initiate RNAi resulting in reduction of the target of interest. The PD effect is closely tied to the overall liver levels which enable efficient and continuous loading into RISC.

Figure 1: Metabolic scheme of sense strand (A) and antisense strand (B). The sense strand contains three GalNAc-moieties attached to a linker which can undergo cleavage of the amide bonds at positions 1 (linker 1 ) thru 3 (linker 3). The antisense strand (Panel B) can undergo metabolism by exonucleases (red) which work on the end of the strand resulting in release of mononucleotides while endonucleases (blue) cleave internally and result in strands of varying lengths. The GalNAc-siRNAs evaluated in these series of studies contain consistent chemical modifications of the 2' ribose including 


\section{DMD-MR-2021-000428}

substitution of a fluoro (green) or O-methyl (black). The GalNAc in the ESC and above classes also contain phophorothioate in place of phophorodiester bonds in the six labeled positions (orange line). The earlier class of GalNAc-siRNA that revusiran and siRNA20 are from called standard template chemistry (STC) contain only two PS bonds at the overhang nucleotides in the antisense strand at the 3 'end.

Figure 2: Mean \pm SD, siRNA5 (Panel A) or siRNA1 (Panel B) duplex plasma concentration-time profiles based on antisense strand (AS) and sense strand (SS) after a single SC administration of varying but similar dose levels in rat, monkey) and human. The data are plotted as the mean and standard deviation (error bars) of $n=4,6$, or 10 datapoints per time-point. The LLOQ for assay was $10 \mathrm{ng} / \mathrm{mL}$ (dashed line). The PK parameters were described using non-compartmental methods. Statistical significance was determined using a 2-tailed, unpaired student t-test with unequal variance. There was no significant difference between AS and SS concentrations at any time-points or in the derived PK parameters. Panel C shows the Pearson correlation coefficient $(r)$ for the AUC $\left(\mathrm{h}^{*} \mu \mathrm{g} / \mathrm{mL}\right)$ values derived from multiple GalNAc-siRNA tested at increasing dose levels in rat (X-axis) and monkey (Y-axis). Panel $\mathrm{D}$ shows the Pearson correlation coefficient $(r)$ for the AUC $\left(h^{*} \mu \mathrm{g} / \mathrm{mL}\right)$ values derived from multiple GalNAc-siRNA tested at increasing dose levels in monkey (X-axis) and human (Y-axis). Panel E depicts the AUC $\left(h^{*} \mu \mathrm{g} / \mathrm{mL}\right)$ values derived for increasing SC dose levels of seven GaINAc-siRNA in human, while Panel $\mathrm{F}$ presents the same data derived in monkey. In these panels siRNA15 is depicted by the blue circle, siRNA2, the red square, siRNA21 the green upward triangle, siRNA5 the purple downward triangle, siRNA6 the orange diamond, siRNA7 the black circle and siRNA13 the brown square. Panel G shows the 


\section{DMD-MR-2021-000428}

concentration time-profile for siRNA 1 (blue circle, closed is following IV administration of $10 \mathrm{mg} / \mathrm{kg}$, open is following SC administration of $10 \mathrm{mg} / \mathrm{kg}$ ), siRNA2 (red square, closed is following IV administration of $10 \mathrm{mg} / \mathrm{kg}$, open is following SC administration of $10 \mathrm{mg} / \mathrm{kg}$ ) and siRNA5 (green triangle, closed is following IV administration of $5 \mathrm{mg} / \mathrm{kg}$, open is following SC administration of $5 \mathrm{mg} / \mathrm{kg}$ ). The table shows the derived PK parameters and the estimated \%F from these studies.

Figure 3: Dose normalized Cmax (A-D) and AUC (E-H) values in male and females livers following SC administration to rat (Panels $A, E), S C$ administration to monkey (Panels B,F). IV administration to rat, (Panels C, G). IV administration to monkey (Panels $\mathrm{D}, \mathrm{H})$. The horizontal line represents the median data point and the vertical lines represent the $90 \%$ confidence intervals in Panels $A, B, E$ and $F$ where multiple dose levels were evaluated following SC administration. For IV dosing only a single dose level was administered and the data points represent the composite mean value for $n=3$ rats or monkeys. In these panels siRNA1 is represented by a blue circle, siRNA2, red triangle, siRNA3 green upwards triangle, siRNA4 purple downwards triangle, siRNA5 orange diamond, siRNA6 black square and siRNA7 brown square. Statistical significance between PK parameters across GalNAc-siRNA between males and females was evaluated using a 2-tailed, unpaired student t-test with unequal variance and there were no statistically significant findings. Panels I. and J. depict the AUC $\left(h^{*} \mu \mathrm{g} / \mathrm{g}\right)$ determined in liver following increasing SC dose levels of siRNA1 through siRNA22 (depicted with varying color circles), in rat (I) and monkey (J). The black line represents the mean linear regression for the dataset while the bluelines represent the slopes between 2 and 0.5 -fold of the mean. 


\section{DMD-MR-2021-000428}

Figure 4: Tissue distribution profile in rats following SC administration of radiolabeled siRNA2 (3 mg/kg), siRNA5 (3 mg/kg) and siRNA20 (10 mg/kg) to rats based on the estimated dose-normalized AUC $\left(h^{*} \mu \mathrm{g} / \mathrm{g}\right)$, Panel A from the QWBA analysis. The QWBA data represents the composite value of $n=3$ animals/time-point. Panel $B$ and $C$ depict the microautoradiography results following $3 \mathrm{mg} / \mathrm{kg}$ administration of siRNA 5 to rats. In these pictures the annotations of $10,11,13$, and 14 , represent the central vein, the centrilobular region, the peri-portal region, the portal vein and the bile duct, respectively.

Figure 5: Metabolite profiles for siRNA2, siRNA5, siRNA6, siRNA9 and siRNA13 in homogenate prepared from monkey livers following SC dosing. The percentage of fulllength (blue) compared with $3^{\prime} \mathrm{N}-1$ (orange) and other active metabolites (green) is derived from the AUC profile either as a pool or discrete time-point samples. In all cases authentic standards were used to determine the quantitative levels of full-length or metabolites and the sum of the individual components was used to derive the percentage of total.

Figure 6: Following SC administration of $3 \mathrm{H}$-siRNA2 in rats a secondary peak of radioactivity was observed starting around $120 \mathrm{~h}$ post dosing. Panel A depicts the primary and secondary radioactivity peaks which already account for the release of tritiated water via deduction. Evaluation of the radiochromatogram from plasma samples taken at the maximum of this secondary peak (Panel B) demonstrate that all of the radioactivity elutes early, where shortmer ( $>3$ but $<7$ oligonucleotides), dimer (2 oligonucleotides) or monomers (single nucleotides and/or nucleosides) elute. There 


\section{DMD-MR-2021-000428}

were no peaks observed at the elution times for siRNA2 $(13.9 \mathrm{~min})$ or the active metabolite AS(N-1)3' (13.5 min)..

Figure 7: Pharmacodynamics were measured following SC administration of $1 \mathrm{mg} / \mathrm{kg}$ siRNA11 to intact female rats (blue) or bile duct cannulated female rats (red) using a commercially available ELISA kit towards the target protein in serum. The y-axis is the relative amount of target remaining and the $x$-axis is the timepoint in hours. Each point represents the mean of three animals per timepoint with the bars representing the standard deviation. A two-tailed, unpaired student t-test with unequal variance was used to evaluate whether there were any statistical differences between the intact and BDC rats at each time-point. There were no statistically significant differences between the groups observed.

Figure 8: The time-profile (x-axis) for concentrations of siRNA17 and two active metabolites were evaluated over 99 days following a single SC administration of 3 $\mathrm{mg} / \mathrm{kg}$ in monkey ( $\mathrm{n}=3)$ in plasma (A, y-axis), liver (B, y-axis), and RISC (C, y-axis). In addition the measurement of target in serum samples was determined using a commercially available ELISA kit to measure pharmacodynamics as a ratio of baseline (pre-dose) levels ( $D, y$-axis). Each point represents the composite mean per time-point.

Figure 9: siRNA7 was administered by single SC injection to mouse (red), monkey (black), and human (blue) at 0.3 (squares) or $3 \mathrm{mg} / \mathrm{kg}$ (circle). The extent and duration of target reduction was evaluated through serum measurements over time. The relative target ( $\mathrm{Y}$-axis) over time ( $\mathrm{X}$-axis) is displayed for $\mathrm{n}=3$ (mouse and monkey), $\mathrm{n}=6$ (human). 


\section{DMD-MR-2021-000428}

Figure 10: Panel A shows the concentration (y-axis) time profile (x-axis) in monkey liver following SC administration of similar single dose levels $(2.5 \mathrm{mg} / \mathrm{kg}$ for revusiran in orange and $3 \mathrm{mg} / \mathrm{kg}$ for vutrisiran in blue. Panel B shows the liver half-life values generated from pharmacokinetic data following single dose administration of revuisrran and vutrisiran to rat and monkey. The composite profile of $n=3$ animals is depicted. Panel $C$ shows the pharmacodynamic activity in monkey $(n=3)$ as relative serum TTR protein level (y-axis) over time in days (x-axis). Revusiran (orange circles) was given SC at $2.5 \mathrm{mg} / \mathrm{kg}$ each day for five days followed by weekly dosing for 4 weeks. Vutrisiran (blue squares) was given as a single $\mathrm{SC}$ injection at $1 \mathrm{mg} / \mathrm{kg}$. Panel D shows the pharmacodynamic activity in human $(n=6)$ as relative serum TTR protein level $(y-$ axis) over time in days (x-axis). Revusiran (orange circles) was given SC at $500 \mathrm{mg}$ each day for five days followed by weekly dosing for 5 weeks. Vutrisiran (blue squares) was given as a single SC injection at $50 \mathrm{mg} / \mathrm{kg}$. 


\section{DMD-MR-2021-000428}

\section{TABLES}

Table 1: Plasma pharmacokinetic parameters from SC dosing of multiple siRNA to male and female rats and monkeys

\begin{tabular}{|c|c|c|c|c|c|c|c|c|c|c|c|}
\hline \multirow{3}{*}{$\begin{array}{c}\text { siRN } \\
A\end{array}$} & \multirow{3}{*}{$\begin{array}{c}\text { Dose } \\
\text { (mg/kg } \\
\text { ) }\end{array}$} & \multicolumn{10}{|c|}{ Plasma Pharmacokinetic Properties Following SC Administration to Monkeys } \\
\hline & & \multicolumn{2}{|c|}{$\mathrm{C}_{\max }(\mathrm{ng} / \mathrm{mL})$} & \multicolumn{2}{|c|}{$\begin{array}{c}\mathrm{AUC}_{\text {last }} \\
\left(\mathrm{h}^{*} \mathrm{ng} / \mathrm{mL}\right)\end{array}$} & \multicolumn{2}{|c|}{$\mathrm{T}_{\max }(\mathrm{h})$} & \multicolumn{2}{|c|}{$\mathrm{T}_{1 / 2} \mathrm{~h}$} & \multicolumn{2}{|c|}{$\%$ dose at $\mathrm{C}_{\max }$} \\
\hline & & M & $\mathrm{F}$ & $\mathrm{M}$ & $\mathrm{F}$ & M & $\mathrm{F}$ & M & $\mathrm{F}$ & M & $\mathrm{F}$ \\
\hline \multirow{3}{*}{1} & 0.3 & 40.6 & 57.6 & 233 & 266 & 1 & 2 & 8.79 & ND & 0.159 & 0.225 \\
\hline & 3 & 634 & 598 & 4700 & 3310 & 2 & 2 & 2.78 & 2.11 & 0.248 & 0.234 \\
\hline & 10 & 2530 & $\begin{array}{c}286 \\
0\end{array}$ & 15700 & $\begin{array}{c}1660 \\
0\end{array}$ & 2 & 2 & 1.96 & 5.84 & 0.297 & 0.335 \\
\hline \multirow{3}{*}{2} & 1 & 89.3 & 173 & 279 & 465 & 1 & 1 & ND & ND & 0.105 & 0.203 \\
\hline & 5 & 857 & 702 & 4820 & 4260 & 2 & 2 & ND & ND & 0.201 & 0.165 \\
\hline & 10 & 2060 & $\begin{array}{c}203 \\
0\end{array}$ & 11300 & $\begin{array}{c}1060 \\
0\end{array}$ & 1 & 2 & 3.36 & ND & 0.242 & 0.238 \\
\hline \multirow{3}{*}{15} & 0.3 & 43.8 & 30.6 & 189 & 162 & 1 & 1 & 2.38 & 2.84 & 0.171 & 0.119 \\
\hline & 1 & 96.2 & 112 & 695 & 686 & 1 & 1 & 3.24 & 3.37 & 0.113 & 0.131 \\
\hline & 3 & 358 & 425 & 2190 & 2680 & 2 & 2 & 2.79 & 2.5 & 0.140 & 0.166 \\
\hline \multirow{3}{*}{3} & 1 & 72.9 & 64 & 112 & 87 & 2 & 2 & ND & ND & $\begin{array}{c}0.085 \\
4\end{array}$ & 0.075 \\
\hline & 5 & 386 & 281 & 1930 & 1690 & 2 & 2 & ND & ND & $\begin{array}{c}0.090 \\
4\end{array}$ & $\begin{array}{c}0.065 \\
8\end{array}$ \\
\hline & 25 & 2560 & $\begin{array}{c}213 \\
0\end{array}$ & 19700 & $\begin{array}{c}1200 \\
0 \\
\end{array}$ & 2 & 1 & 3.79 & 6.13 & 0.120 & $\begin{array}{c}0.099 \\
8\end{array}$ \\
\hline \multirow{4}{*}{4} & 0.3 & 82.1 & 86.8 & 198 & 204 & 1 & 1 & ND & ND & 0.321 & 0.339 \\
\hline & 1 & 245 & 311 & 843 & 949 & 1 & 0.5 & 2.55 & 1.52 & 0.287 & 0.364 \\
\hline & 5 & 1140 & $\begin{array}{c}125 \\
0\end{array}$ & 5610 & 5850 & 1 & 1 & 3.04 & 1.81 & 0.268 & 0.293 \\
\hline & 20 & 6770 & $\begin{array}{c}448 \\
0\end{array}$ & 44000 & $\begin{array}{c}3800 \\
0\end{array}$ & 2 & 4 & 8.49 & ND & 0.396 & 0.263 \\
\hline \multirow{4}{*}{5} & 0.1 & 13.8 & 13.2 & 168 & 22.5 & 4 & 1 & ND & ND & 0.161 & 0.155 \\
\hline & 1 & 181 & 129 & 12200 & 1140 & 2 & 2 & ND & 9.94 & 0.212 & 0.151 \\
\hline & 5 & 1160 & 985 & 5590 & 4240 & 2 & 2 & ND & ND & 0.272 & 0.231 \\
\hline & 10 & 1570 & $\begin{array}{c}225 \\
0\end{array}$ & 12100 & $\begin{array}{c}1070 \\
0 \\
\end{array}$ & 2 & 2 & 3.58 & ND & 0.184 & 0.263 \\
\hline \multirow{4}{*}{6} & 0.3 & 42.2 & 37.6 & 119 & 127 & 0.5 & 2 & 6.22 & ND & 0.165 & 0.147 \\
\hline & 1 & 90.6 & 99.7 & 586 & 662 & 4 & 4 & ND & ND & 0.106 & 0.117 \\
\hline & 3 & 549 & 299 & 3140 & 1600 & 4 & 2 & ND & ND & 0.214 & 0.117 \\
\hline & 30 & 7760 & $\begin{array}{c}692 \\
0\end{array}$ & 63100 & $\begin{array}{c}6610 \\
0\end{array}$ & 2 & 4 & 2.7 & ND & 0.303 & 0.270 \\
\hline \multirow{3}{*}{$\begin{array}{c}\operatorname{siRN} \\
A\end{array}$} & \multirow{3}{*}{$\begin{array}{c}\text { Dose } \\
\text { (mg/kg } \\
\text { ) }\end{array}$} & \multicolumn{10}{|c|}{ Plasma Pharmacokinetic Properties Following SC Administration to Rats } \\
\hline & & \multicolumn{2}{|c|}{$\mathrm{C}_{\max }(\mathrm{ng} / \mathrm{mL})$} & \multicolumn{2}{|c|}{$\begin{array}{c}\mathrm{AUC}_{\text {last }} \\
\left(\mathrm{h}^{*} \mathrm{ng} / \mathrm{mL}\right)\end{array}$} & \multicolumn{2}{|c|}{$\mathrm{T}_{\max }(\mathrm{h})$} & \multicolumn{2}{|c|}{$\mathrm{T}_{1 / 2} \mathrm{~h}$} & \multicolumn{2}{|c|}{$\%$ dose at $\mathrm{C}_{\max }$} \\
\hline & & M & $\mathrm{F}$ & $\mathrm{M}$ & $\mathrm{F}$ & M & $\mathrm{F}$ & M & $\mathrm{F}$ & M & $\mathrm{F}$ \\
\hline 1 & 0.3 & 34.7 & 29.1 & 39.6 & 16.8 & 1 & 1 & ND & ND & 0.453 & 0.381 \\
\hline
\end{tabular}


DMD-MR-2021-000428

\begin{tabular}{|c|c|c|c|c|c|c|c|c|c|c|c|}
\hline & 1 & 90.5 & 92.4 & 174 & 171 & 1 & 1 & ND & ND & 0.355 & 0.363 \\
\hline & 3 & 290 & 301 & 494 & 479 & 1 & 1 & ND & ND & 0.379 & 0.395 \\
\hline & 10 & 1430 & $\begin{array}{c}106 \\
0\end{array}$ & 3740 & 2120 & 1 & 1 & ND & 1.38 & 0.561 & 0.415 \\
\hline \multirow{6}{*}{2} & 1 & 98 & 112 & 141 & 158 & $\begin{array}{c}0.2 \\
5\end{array}$ & $\begin{array}{c}0.2 \\
5\end{array}$ & ND & 2.54 & 0.384 & 0.44 \\
\hline & 1 & 102 & 113 & 254 & 164 & 2 & 1 & ND & ND & 0.401 & 0.442 \\
\hline & 5 & 466 & 386 & 1400 & 1110 & 0.5 & $\begin{array}{c}0.2 \\
5\end{array}$ & 3.14 & 1.66 & 0.365 & 0.303 \\
\hline & 5 & 568 & 455 & 1580 & 1240 & 2 & 1 & ND & ND & 0.445 & 0.357 \\
\hline & 10 & 976 & $\begin{array}{c}117 \\
0\end{array}$ & 2390 & 2980 & $\begin{array}{c}0.2 \\
5\end{array}$ & 2 & 3.31 & ND & 0.383 & 0.458 \\
\hline & 10 & 1120 & $\begin{array}{c}155 \\
0\end{array}$ & 4150 & 3820 & 2 & 2 & 6.33 & ND & 0.441 & 0.607 \\
\hline \multirow{3}{*}{3} & 2.5 & 230 & 285 & 320 & 371 & 0.5 & 0.5 & ND & ND & 0.36 & 0.447 \\
\hline & 5 & 514 & 639 & 934 & 762 & 1 & 0.5 & ND & ND & 0.403 & 0.502 \\
\hline & 25 & 4450 & $\begin{array}{c}398 \\
0\end{array}$ & 10300 & 7080 & 1 & 1 & 1.43 & ND & 0.698 & 0.625 \\
\hline \multirow{4}{*}{4} & 0.3 & 71.4 & 93.9 & 101 & 111 & 0.5 & 0.5 & ND & ND & 0.933 & 1.23 \\
\hline & 1 & 312 & 207 & 415 & 262 & 0.5 & 0.5 & $\begin{array}{c}0.96 \\
9\end{array}$ & ND & 1.22 & 0.814 \\
\hline & 5 & 943 & 943 & 1840 & 2020 & $\begin{array}{c}0.2 \\
5\end{array}$ & 0.5 & $\begin{array}{c}0.93 \\
1\end{array}$ & 0.83 & 0.739 & 0.741 \\
\hline & 20 & 4130 & $\begin{array}{c}499 \\
0\end{array}$ & 16200 & $\begin{array}{c}1060 \\
0\end{array}$ & 0.5 & 1 & ND & 1.02 & 0.809 & 0.981 \\
\hline \multirow{4}{*}{5} & 0.1 & 21.7 & 22 & 15 & 14.2 & 0.5 & 0.5 & ND & 0.987 & 0.849 & 0.865 \\
\hline & 1 & 271 & 335 & 381 & 498 & 0.5 & 0.5 & 1.03 & ND & 1.06 & 1.32 \\
\hline & 5 & 1010 & 874 & 1820 & 2020 & 0.5 & 1 & 1.05 & 1.11 & 0.793 & 0.687 \\
\hline & 10 & 2200 & $\begin{array}{c}243 \\
0\end{array}$ & 4770 & 4620 & 1 & 0.5 & $\begin{array}{c}0.91 \\
4\end{array}$ & ND & 0.864 & 0.955 \\
\hline
\end{tabular}

Table 2: Estimated \%F from traditional IV bolus or 1-hr infusions compared with SC administration

\begin{tabular}{|c|c|c|c|}
\hline \multirow{2}{*}{ siRNA } & \multicolumn{3}{|c|}{ DN AUC $_{\text {last }}$ SC / DN AUC } \\
\cline { 2 - 4 } & $\begin{array}{c}\text { Bolus or } \\
\text { Infusion }\end{array}$ & Rat & Monkey \\
\hline siRNA1 & Bolus & 15 & ND \\
\hline \multirow{2}{*}{ siRNA2 } & Bolus & 24 & 37 \\
\cline { 2 - 4 } & $1-$ hr Infusion & 225 & ND \\
\hline siRNA3 & Bolus & 25 & 23 \\
\hline siRNA4 & Bolus & 23 & 31 \\
\hline siRNA5 & Bolus & 31 & 50 \\
\hline \multirow{2}{*}{ siRNA6 } & Bolus & 13 & 14 \\
\cline { 2 - 4 } & $1-h r$ Infusion & 104 & ND \\
\hline siRNA 9 & $1-h r$ Infusion & 148 & 115 \\
\hline
\end{tabular}


DMD Fast Forward. Published on June 21, 2021 as DOI: 10.1124/dmd.121.000428

This article has not been copyedited and formatted. The final version may differ from this version.

DMD-MR-2021-000428

\begin{tabular}{|c|c|c|c|}
\hline siRNA18 & 1-hr Infusion & 32 & 79 \\
\hline siRNA22 & Bolus & 37 & 51 \\
\hline
\end{tabular}




\section{DMD-MR-2021-000428}

Table 3: Representative ratio of liver / plasma following SC administration decreases with increasing dose in rat and monkey

\begin{tabular}{|c|c|c|c|c|}
\hline \multirow{2}{*}{ siRNA } & \multicolumn{2}{|c|}{ Dose (mg/kg) } & \multicolumn{2}{|c|}{$\mathrm{AUC}_{\text {last }}$ liver / $\mathrm{AUC}_{\text {last }}$ plasma } \\
\hline & rat & monkey & rat & monkey \\
\hline \multirow{4}{*}{ siRNA1 } & 0.3 & 0.3 & 4905 & 6110 \\
\hline & 1 & ND & 3967 & ND \\
\hline & 3 & 3 & 4482 & 2455 \\
\hline & 10 & 10 & 1955 & 4123 \\
\hline \multirow{3}{*}{ siRNA2 } & 1 & 1 & 3206 & 7758 \\
\hline & 5 & 5 & 4125 & 3473 \\
\hline & 10 & 10 & 2215 & 4142 \\
\hline \multirow{3}{*}{ siRNA3 } & 2.5 & 1 & 3233 & 12558 \\
\hline & 5 & 5 & 1648 & 4729 \\
\hline & 25 & 25 & ND & 2634 \\
\hline \multirow{4}{*}{ siRNA4 } & 0.3 & 0.3 & 14476 & 12169 \\
\hline & 1 & 1 & 19816 & 11927 \\
\hline & 5 & 5 & 16621 & 6628 \\
\hline & 20 & 20 & ND & 2382 \\
\hline \multirow{4}{*}{ siRNA5 } & 0.1 & 0.1 & 6887 & 5749 \\
\hline & 1 & 1 & 2776 & ND \\
\hline & 5 & 5 & 2899 & 7337 \\
\hline & 10 & 10 & 1848 & 6063 \\
\hline \multirow{4}{*}{ siRNA6 } & \multirow{4}{*}{$\begin{array}{c}\text { Not } \\
\text { determined } \\
\text { (ND) }\end{array}$} & 0.3 & ND & 21098 \\
\hline & & 1 & ND & 11927 \\
\hline & & 3 & ND & 6628 \\
\hline & & 30 & ND & 2382 \\
\hline
\end{tabular}




\section{DMD-MR-2021-000428}

Table 4: In Vitro GalNAc and linker metabolism for siRNA8 in cultured hepatocytes

\begin{tabular}{|c|c|c|c|}
\hline \multirow{2}{*}{ Analyte } & \multicolumn{3}{|c|}{ \% of Total AUC siRNA8 sense strand metabolite } \\
& Rrofile & \multicolumn{2}{|c|}{} \\
\cline { 2 - 4 } & Rat & Monkey & Human \\
\hline - 3 GalNAc & Not detected & 48.7 & 45.0 \\
\hline - 3 GalNAc, -1linker1 & Not detected & 20.3 & 27.5 \\
\hline - 3 GalNAc, -1linker1, - 1linker2 & Not detected & 5.2 & Not detected \\
\hline - 3 GalNAc, -2linker1 & Not detected & 12.2 & 14.9 \\
\hline - 3 GalNAc, -2linker1, - 1linker2 & Not detected & 3.6 & Not detected \\
\hline - 3 GalNAc, -3linker1, & 99.5 & 8.5 & 11.0 \\
\hline - 3 GalNAc, -3linker1, - 1linker2 & Not detected & 1.6 & Not detected \\
\hline Full length siRNA8 & 66.6 & 71.0 & 74.9 \\
\hline AS(N-1)3' siRNA8 & 29.7 & 21.2 & 15.9 \\
\hline
\end{tabular}




\section{DMD-MR-2021-000428}

Table 5: Pharmacological activity assessment for siRNA2 metabolites (Transfected) in plated HEP3B

\begin{tabular}{|c|c|}
\hline Designation (AS) & $\begin{array}{l}\% \text { of target mRNA baseline } \\
\text { remaining }\end{array}$ \\
\hline Parent & 16.4 \\
\hline $3^{\prime} \mathrm{N}-1$ & 10.3 \\
\hline $3^{\prime} \mathrm{N}-2$ & 11.4 \\
\hline $3^{\prime} \mathrm{N}-3$ & 13.5 \\
\hline $3^{\prime} N-4$ & 15.9 \\
\hline $3^{\prime} N-5$ & 12.2 \\
\hline $3^{\prime} \mathrm{N}-6$ & 47.7 \\
\hline $3^{\prime} \mathrm{N}-7$ & 81.1 \\
\hline $3^{\prime} \mathrm{N}-8$ & 95.7 \\
\hline $5^{\prime} \mathrm{N}-1$ & 78.6 \\
\hline $5^{\prime} \mathrm{N}-2$ & 20.7 \\
\hline $5^{\prime} \mathrm{N}-3$ & 89.0 \\
\hline $5 ' \mathrm{~N}-4$ & 78.5 \\
\hline $5^{\prime} \mathrm{N}-5$ & 95.2 \\
\hline $5^{\prime} \mathrm{N}-6$ & 100 \\
\hline $5^{\prime} N-7$ & 100 \\
\hline $5^{\prime} \mathrm{N}-8$ & 100 \\
\hline
\end{tabular}




\section{DMD-MR-2021-000428}

Table 6: Cross species renal excretion profiles as a percent of the total dose

\begin{tabular}{|c|c|c|c|}
\hline Species & lumasiran & givosiran & vutrisiran \\
\hline Rat & $9 \%$ & $11 \%$ & $9 \%$ \\
\hline Monkey & $15-25 \%$ & $19 \%$ & $11-24 \%$ \\
\hline Human & $8-25 \%$ & $3-17 \%$ & $10-25 \%$ \\
\hline
\end{tabular}

Table 7: Summary of PK/PD and urinary excretion in rats with and without 5/6 nephrectomy

\begin{tabular}{|c|c|c|c|c|c|c|c|}
\hline \multirow[b]{2}{*}{ siRNA } & \multirow[b]{2}{*}{ Condition } & \multicolumn{2}{|c|}{ Plasma } & \multicolumn{2}{|c|}{ Liver } & \multirow[b]{2}{*}{$\begin{array}{l}\% \text { Urine } \\
\text { Excretion }\end{array}$} & \multirow{2}{*}{$\begin{array}{l}\% \text { mRNA } \\
\text { Reduction }\end{array}$} \\
\hline & & $\begin{array}{c}\mathrm{C}_{\max } \\
(\mu \mathrm{g} / \mathrm{mL})\end{array}$ & $\begin{array}{c}\mathrm{AUC}_{\text {last }} \\
\left(\mathrm{h}^{*} \mathrm{\mu g} / \mathrm{mL}\right)\end{array}$ & $\begin{array}{c}C_{\max } \\
(\mu g / g)\end{array}$ & $\begin{array}{c}\text { AUC }_{\text {last }} \\
\left(h^{*} \mu \mathrm{g} / \mathrm{g}\right)\end{array}$ & & \\
\hline \multirow{2}{*}{ siRNA12 } & Sham & 0.161 & 0.501 & 14.5 & ND & $\begin{array}{c}42 \\
\mathrm{ng} / \mathrm{mL}^{*}\end{array}$ & 74 \\
\hline & Nephrectomy & 0.131 & 0.606 & 14.6 & ND & $\begin{array}{c}13 \\
\mathrm{ng} / \mathrm{mL}^{*}\end{array}$ & 73 \\
\hline \multirow{2}{*}{ siRNA14 } & Sham & 0.048 & 0.198 & 8.0 & 752 & 5 & 70 \\
\hline & Nephrectomy & 0.034 & 0.139 & 5.8 & 765 & 0.74 & 70 \\
\hline \multirow{2}{*}{ siRNA5 } & Sham & 0.080 & 0.303 & 11.9 & ND & 3.2 & 70 \\
\hline & Nephrectomy & 0.081 & 0.300 & 14.7 & ND & 1.1 & 75 \\
\hline
\end{tabular}

* - Total urine volume not available for normalization 
DMD Fast Forward. Published on June 21, 2021 as DOI: 10.1124/dmd.121.000428

This article has not been copyedited and formatted. The final version may differ from this version.

A.

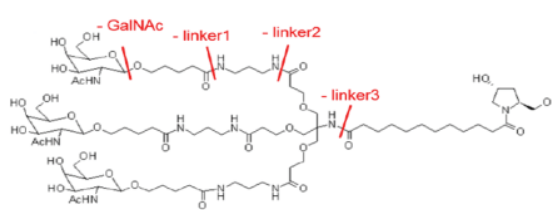

B.

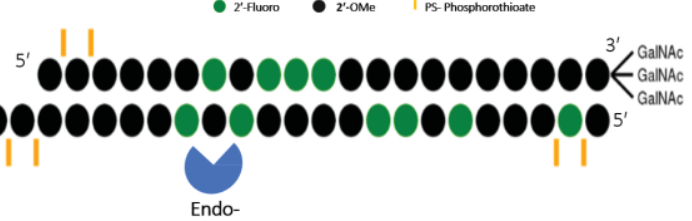

Figure 1 
A.

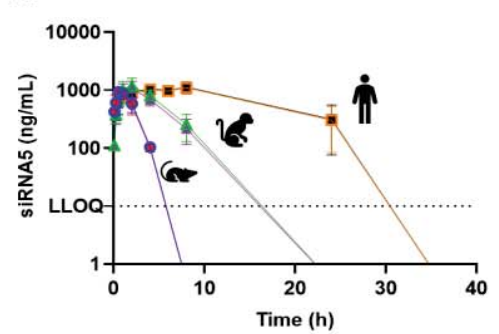

\begin{tabular}{|c|c|c|c|c|c|c|}
\hline \multirow{2}{*}{ Parameter } & \multicolumn{2}{|c|}{ rat } & \multicolumn{2}{c|}{ monkey } & \multicolumn{2}{c|}{ human } \\
\cline { 2 - 8 } & AS & SS & AS & SS & AS & SS \\
\hline $\mathrm{T}_{\max }(\mathrm{h})$ & 0.5 & 0.5 & 2 & 2 & 8 & 8 \\
\hline $\mathrm{C}_{\max }(\mathrm{ng} / \mathrm{mL})$ & 936 & 902 & 1180 & 1070 & 1100 & 1130 \\
\hline $\begin{array}{c}\mathrm{A} \mathrm{NC} \\
\left(\mathrm{h}^{*} \mathrm{ng} / \mathrm{mL}\right)\end{array}$ & 2170 & 2060 & 5780 & 5120 & 18,670 & 19,200 \\
\hline
\end{tabular}

C.

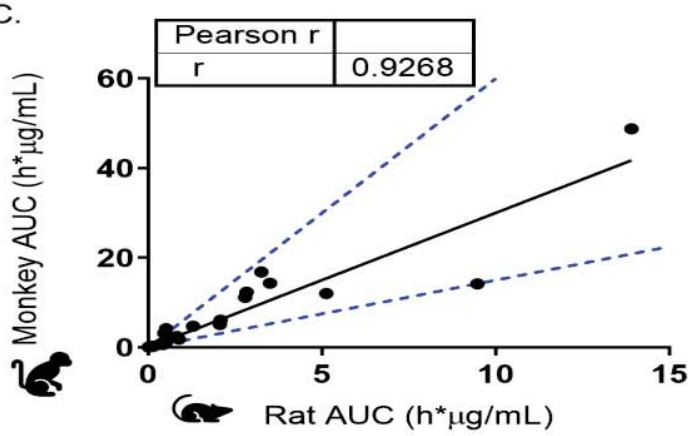

E.

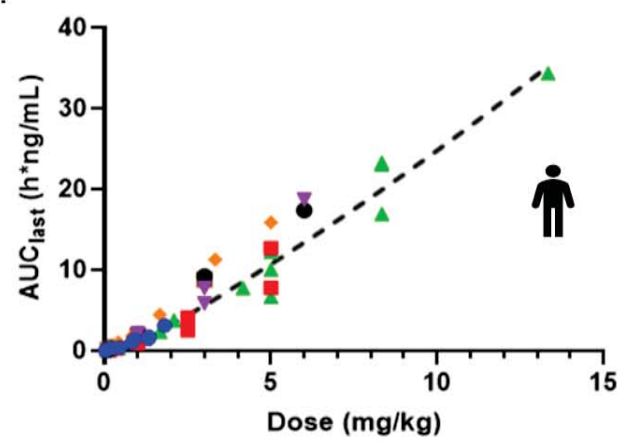

G.

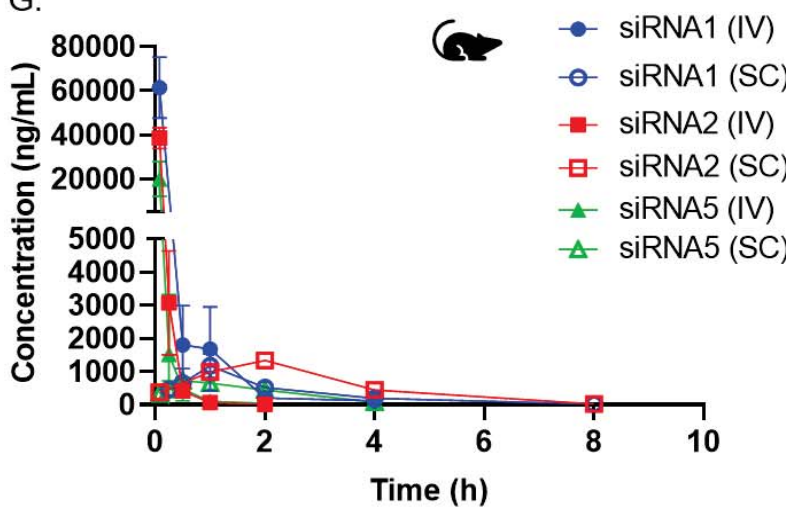

B.

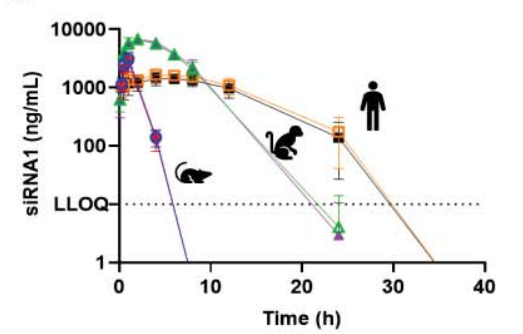

- rat $15 \mathrm{mg} / \mathrm{kg} \mathrm{AS}$

- rat $15 \mathrm{mg} / \mathrm{kg} \mathrm{SS}$

$\triangle$ monkey $25 \mathrm{mg} / \mathrm{kg}$ AS

- monkey $25 \mathrm{mg} / \mathrm{kg} \mathrm{SS}$

- human $6 \mathrm{mg} / \mathrm{kg} \mathrm{AS}$

- human $6 \mathrm{mg} / \mathrm{kg}$ SS

\begin{tabular}{|c|c|c|c|c|c|c|}
\hline \multirow{2}{*}{ Parameter } & \multicolumn{2}{|c|}{ rat } & \multicolumn{2}{c|}{ monkey } & \multicolumn{2}{c|}{ human } \\
\cline { 2 - 8 } & AS & SS & AS & SS & AS & SS \\
\hline$T_{\max }(\mathrm{h})$ & 1 & 1 & 2 & 2 & 4 & 4 \\
\hline $\mathrm{C}_{\max }(\mathrm{ng} / \mathrm{mL})$ & 3110 & 2830 & 6710 & 6740 & 1670 & 1450 \\
\hline $\begin{array}{c}\text { AUC } \\
\left(\mathrm{h}^{*} \mathrm{ng} / \mathrm{mL}\right)\end{array}$ & 7160 & 6460 & 53,700 & 57,100 & 24,600 & 21,600 \\
\hline
\end{tabular}
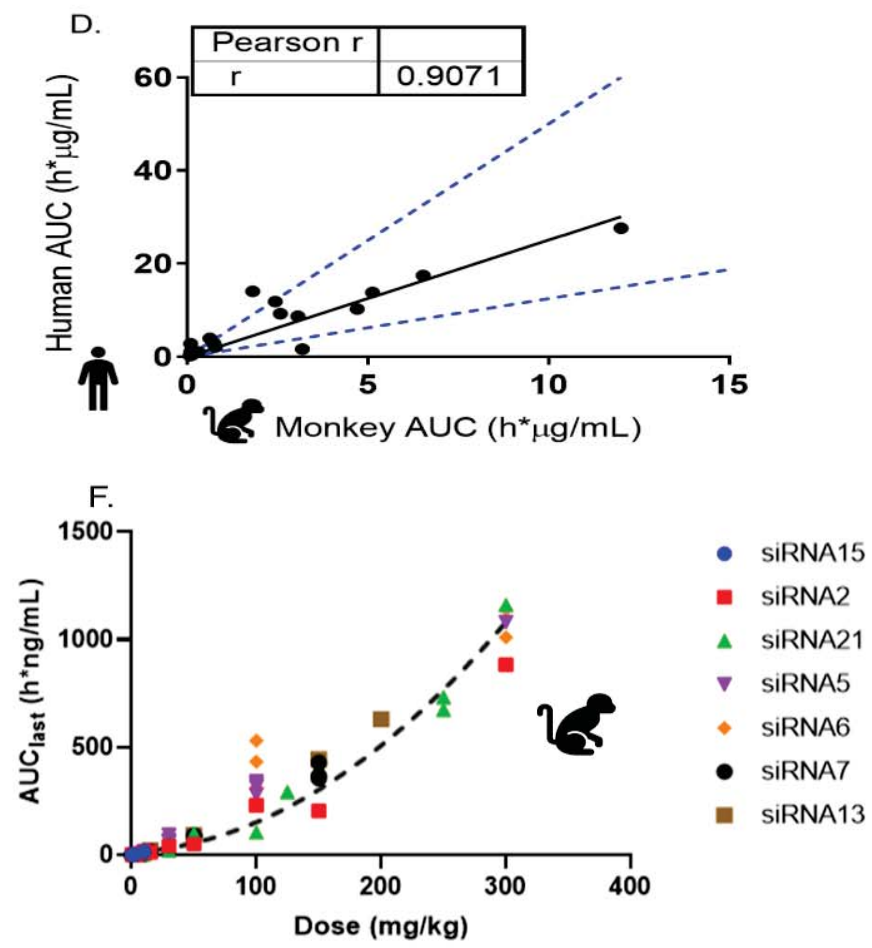

\begin{tabular}{|c|c|c|c|}
\hline \multirow{2}{*}{ siRNA } & \multicolumn{2}{|c|}{ AUC $\left(h^{*}\right.$ ng/mL) } & \multirow{2}{*}{$\%$ FF } \\
\cline { 2 - 4 } & IV & SC & \\
\hline $1(10 \mathrm{mg} / \mathrm{kg})$ & 10000 & 2640 & 26.4 \\
\hline $2(10 \mathrm{mg} / \mathrm{kg})$ & 5670 & 3230 & 57.0 \\
\hline $5(5 \mathrm{mg} / \mathrm{kg})$ & 3090 & 1650 & 53.4 \\
\hline
\end{tabular}

\section{Figure 2}


A.

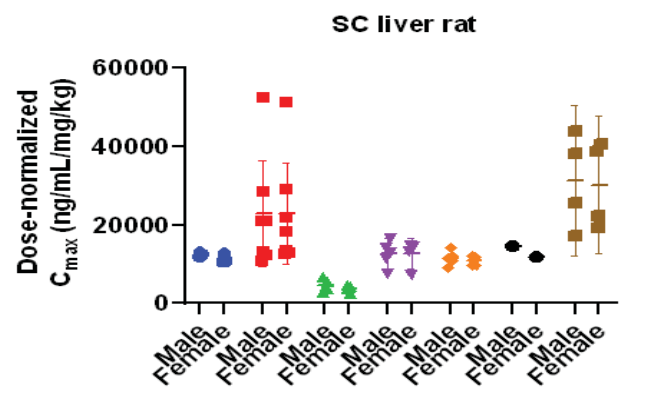

- siRnA1

- sirna2

- SIRNA3

- SIRNA4

- siRNA5

- siRnA6

- siRNA7

c.

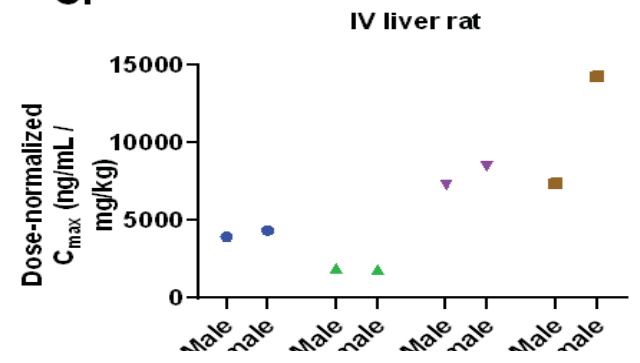

E.

Sc liver rat

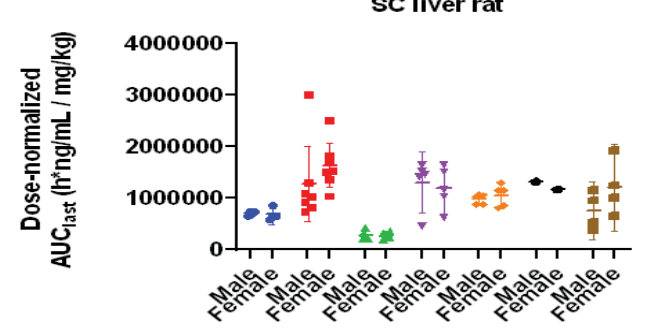

G.

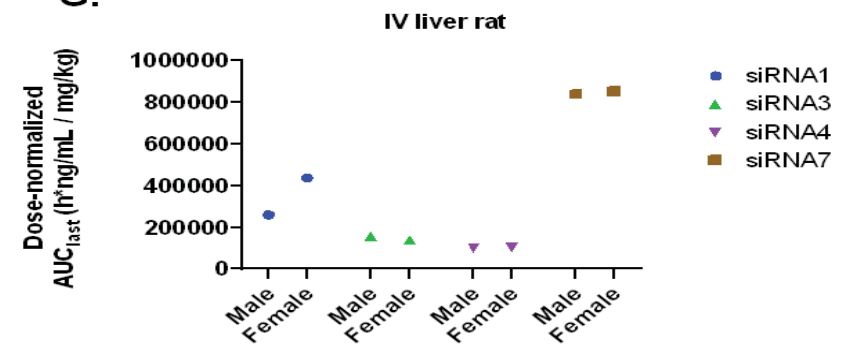

I.

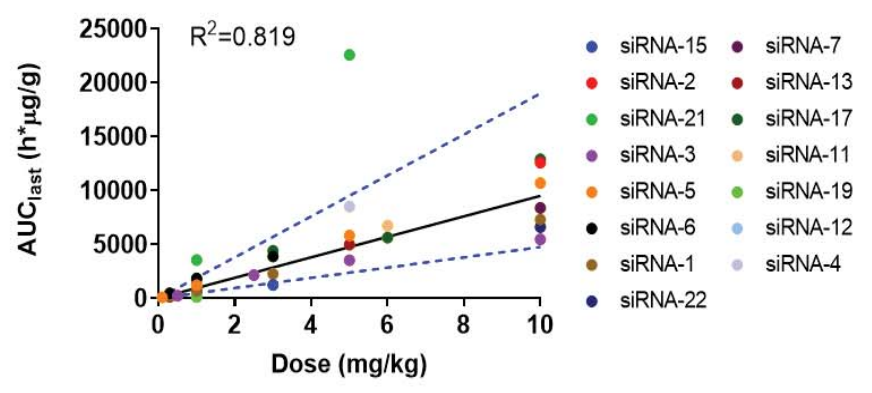

B.

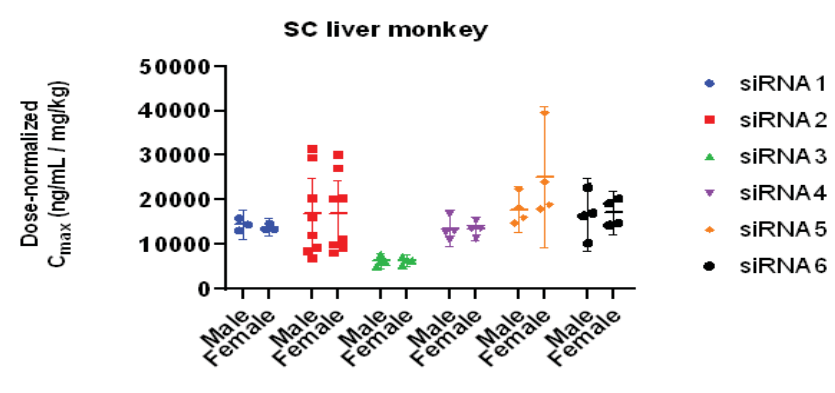

D.

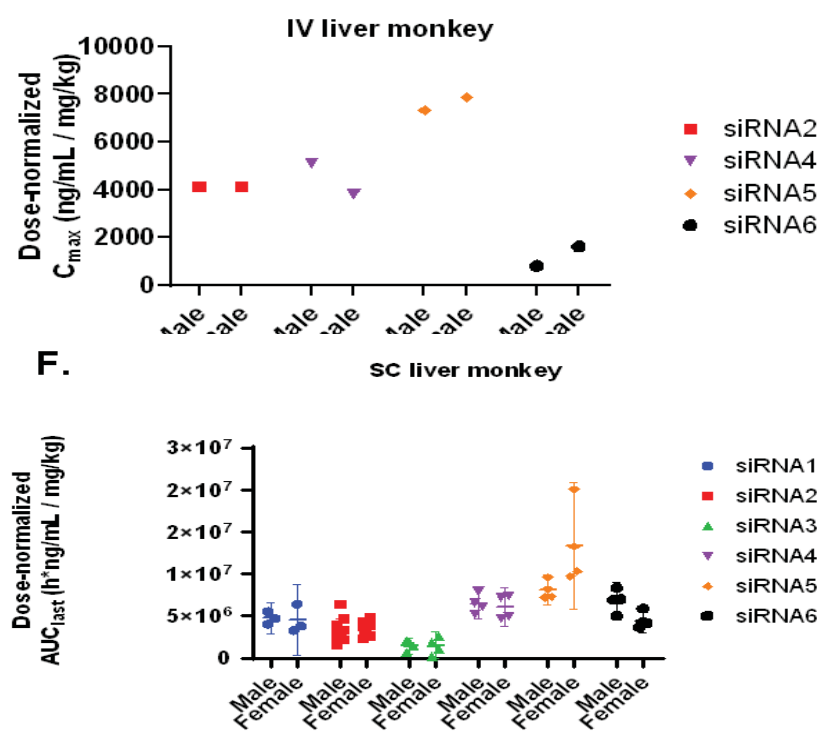

H.

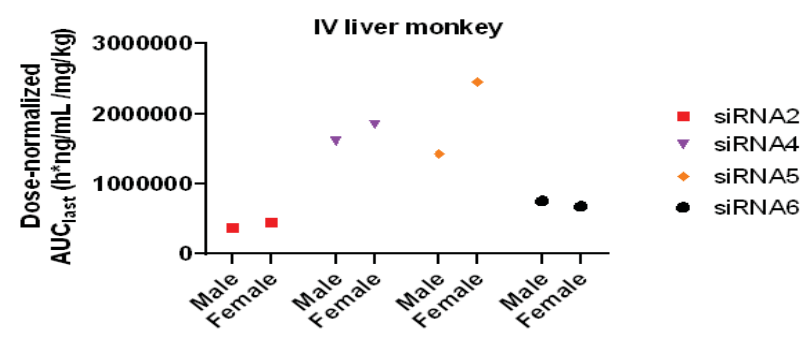

J.

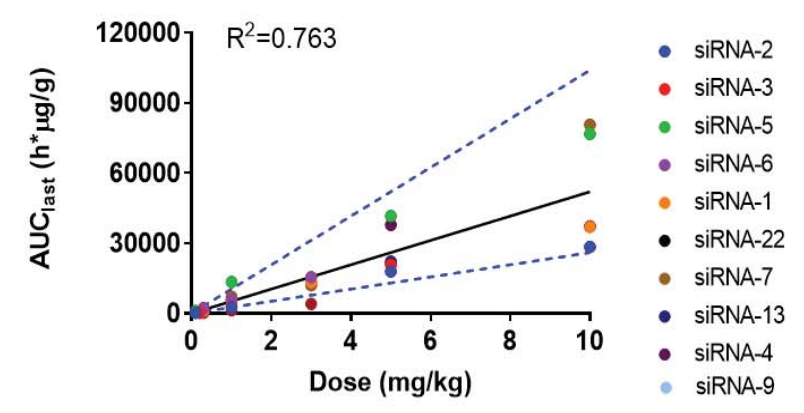

Figure 3 
A.

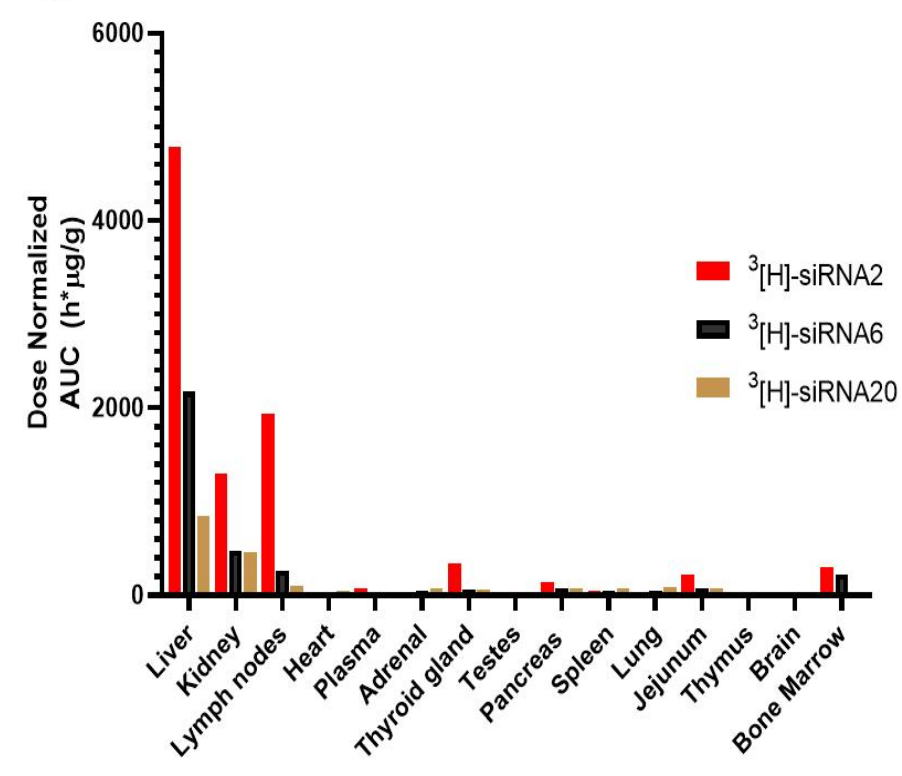

B.

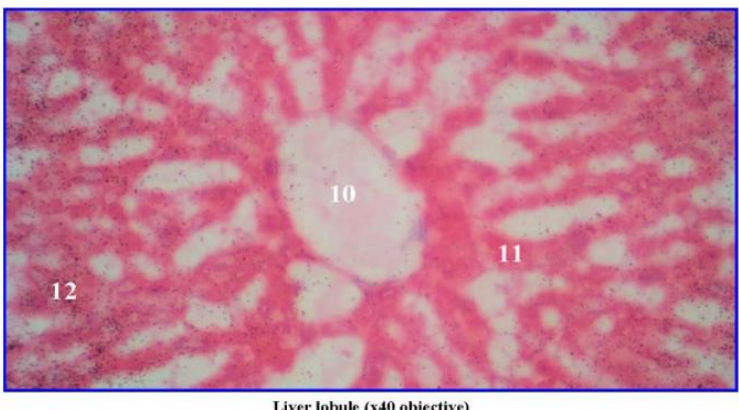

C.

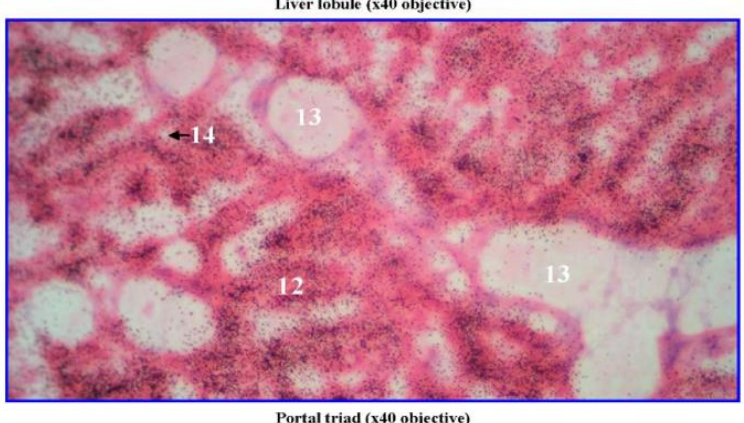

Figure 4 


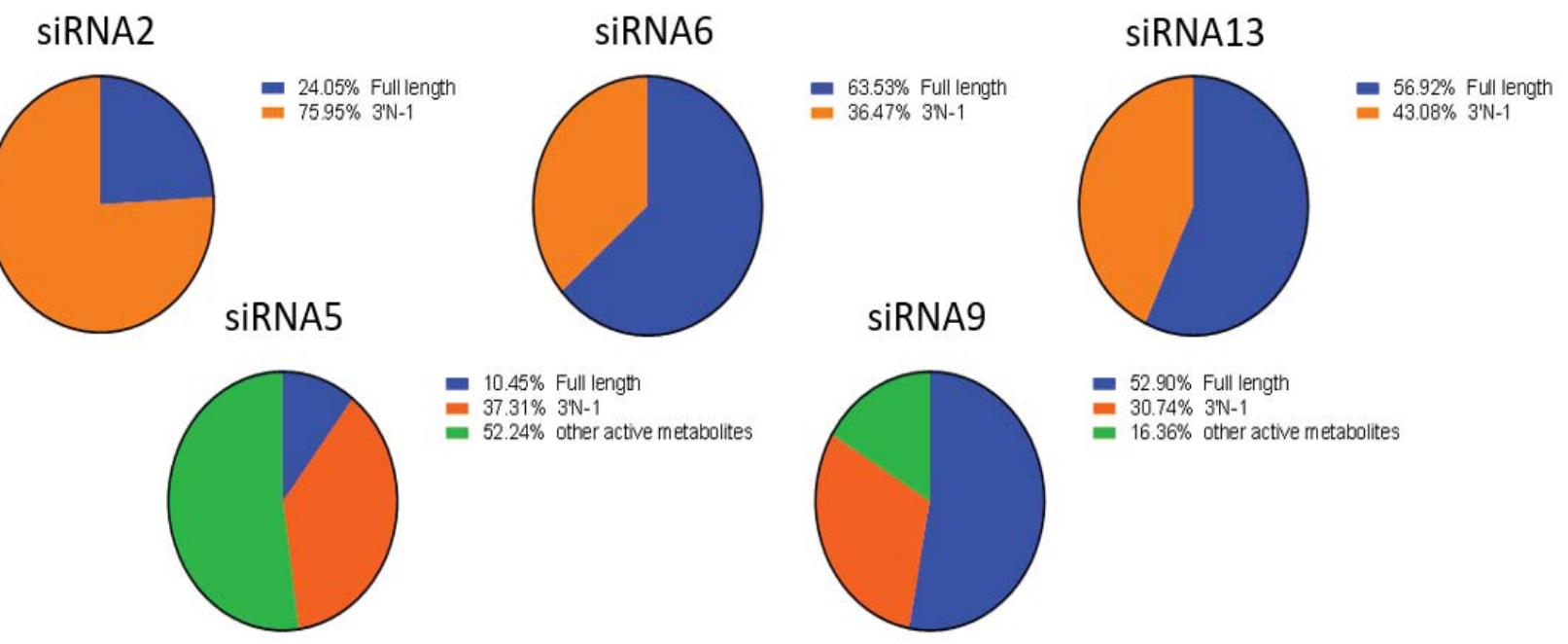

Figure 5 
A.

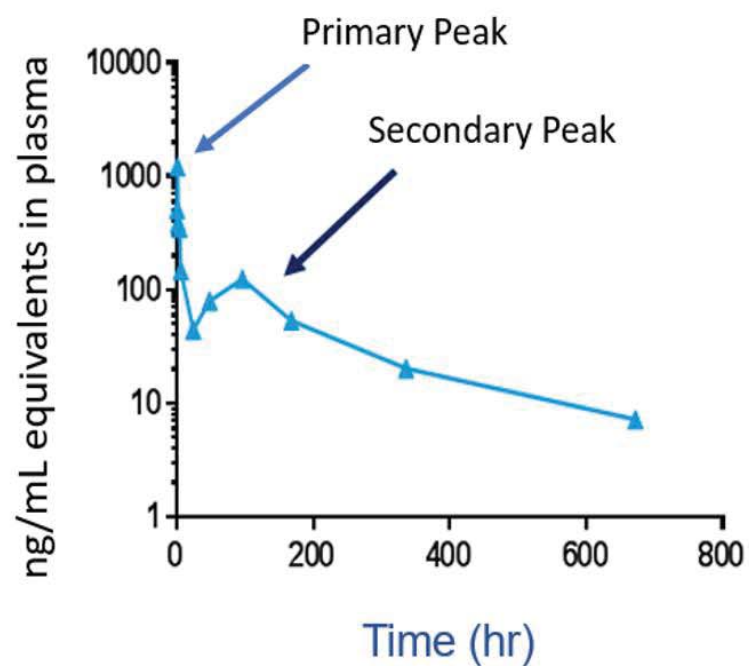

B.

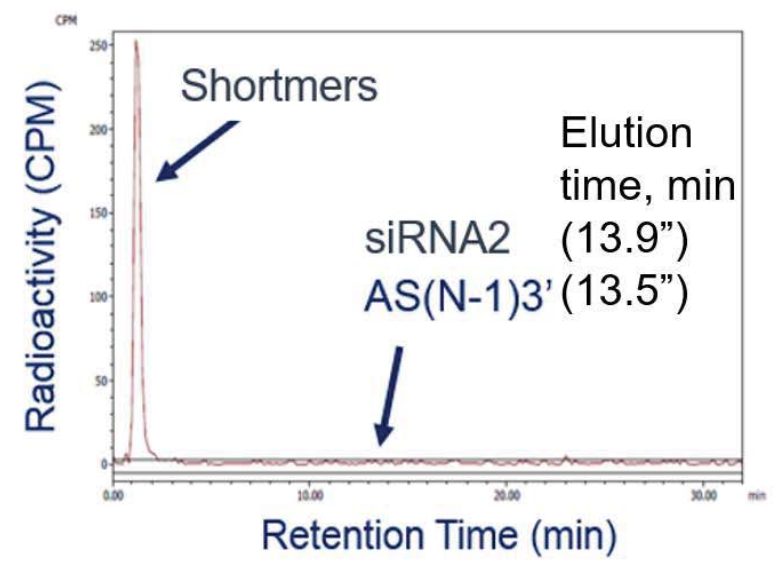

Figure 6 


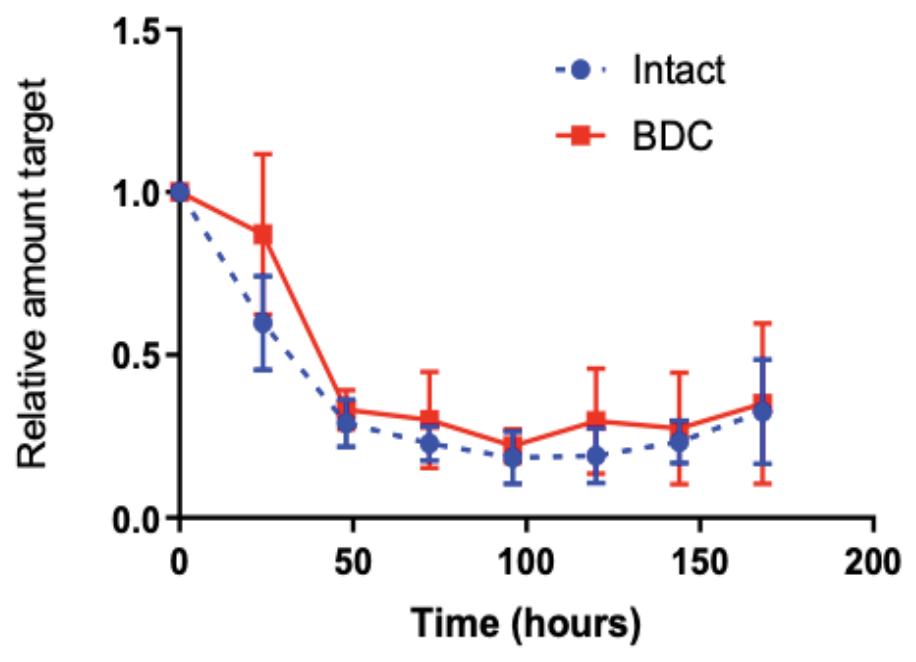

Figure 7 
A.

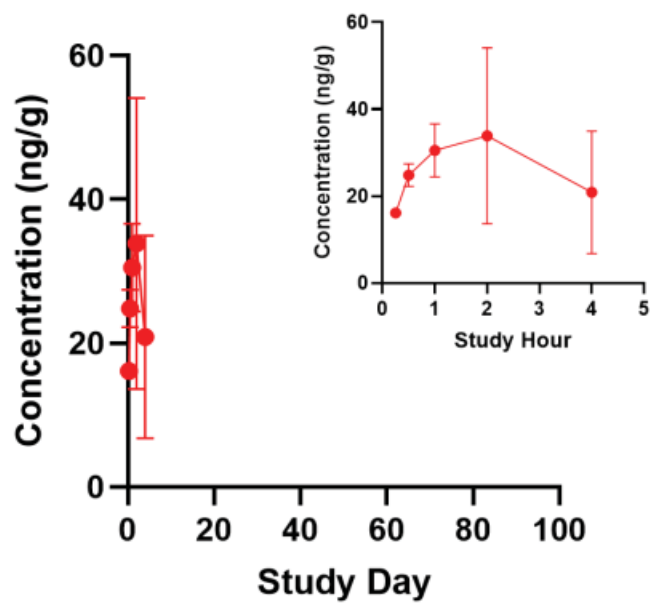

B.

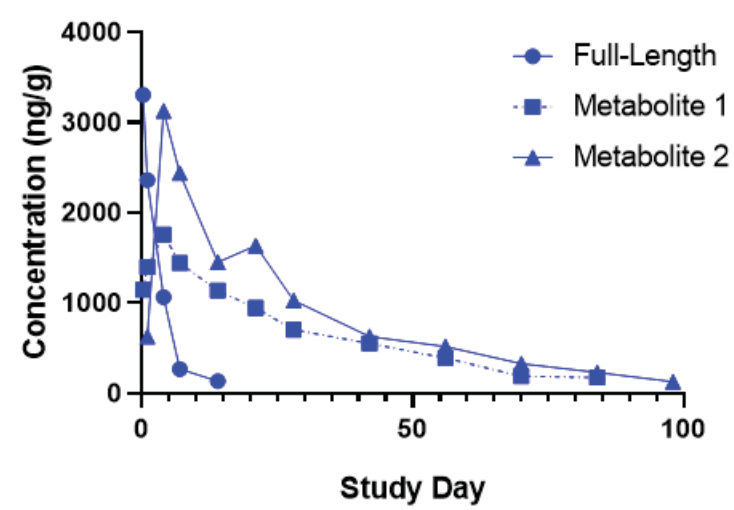

C.

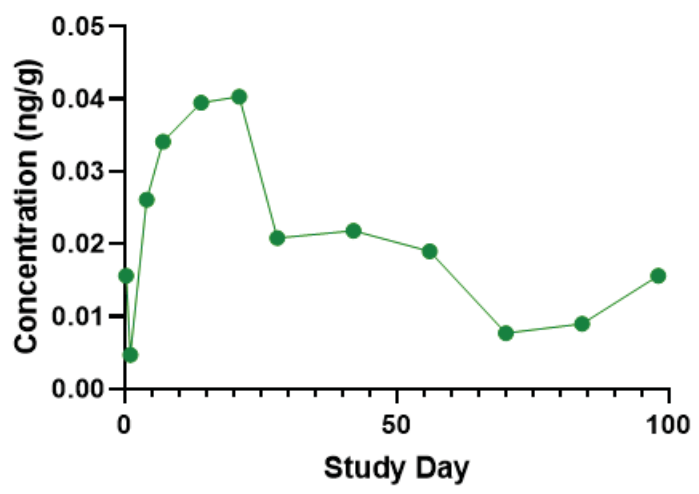

D.

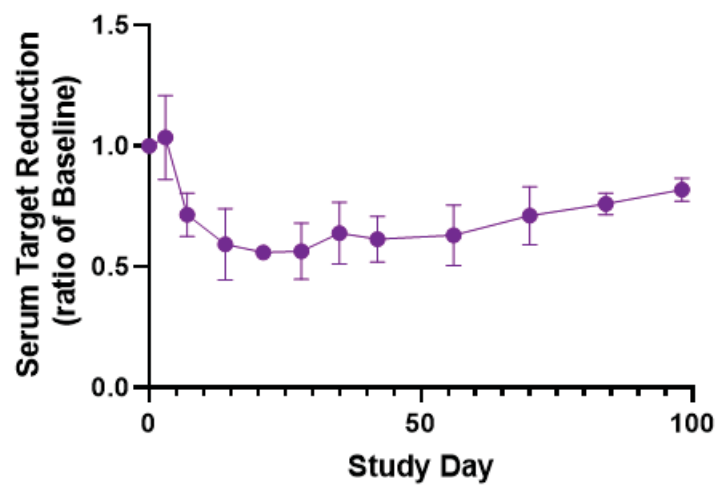

\section{Figure 8}




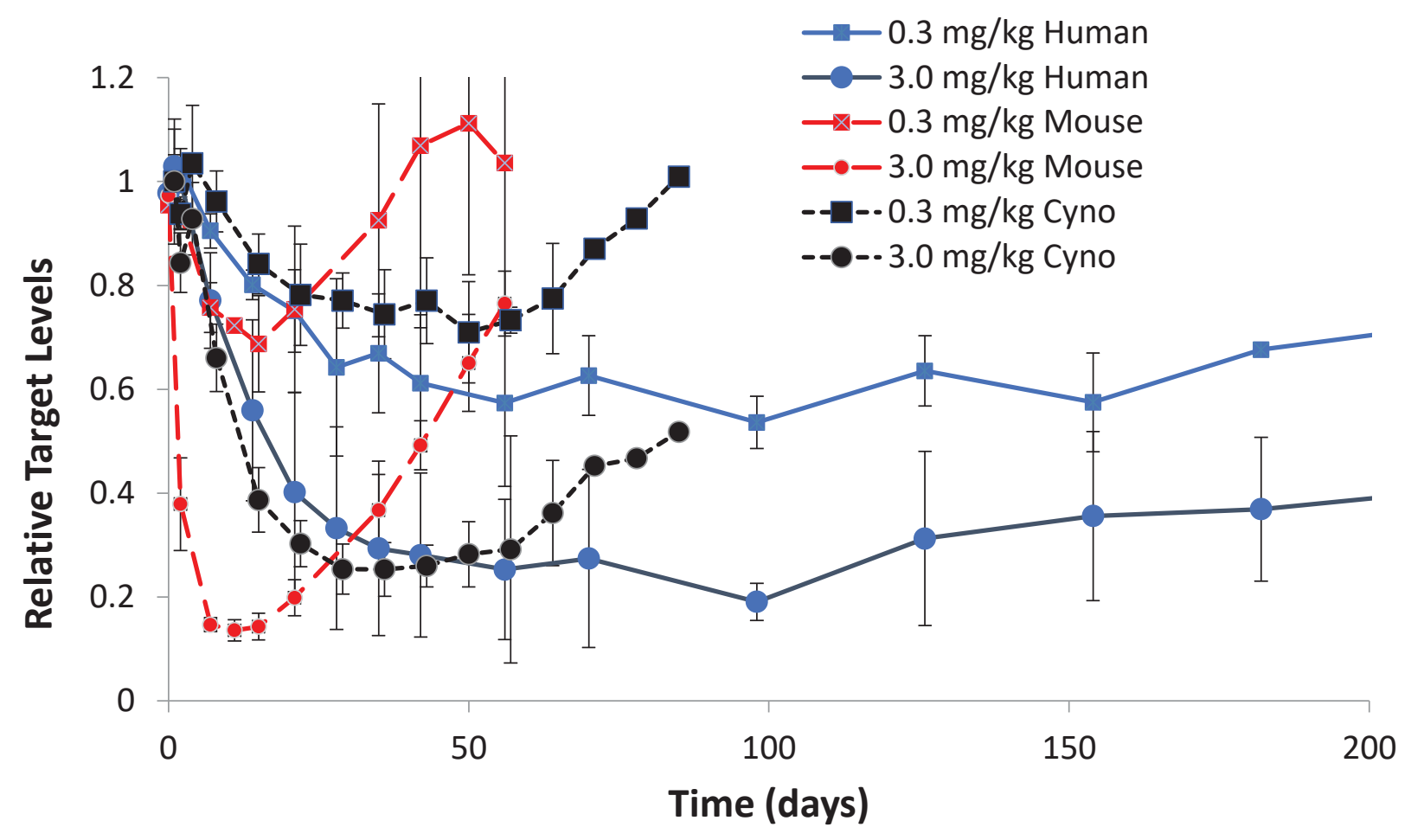

Figure 9 
A.

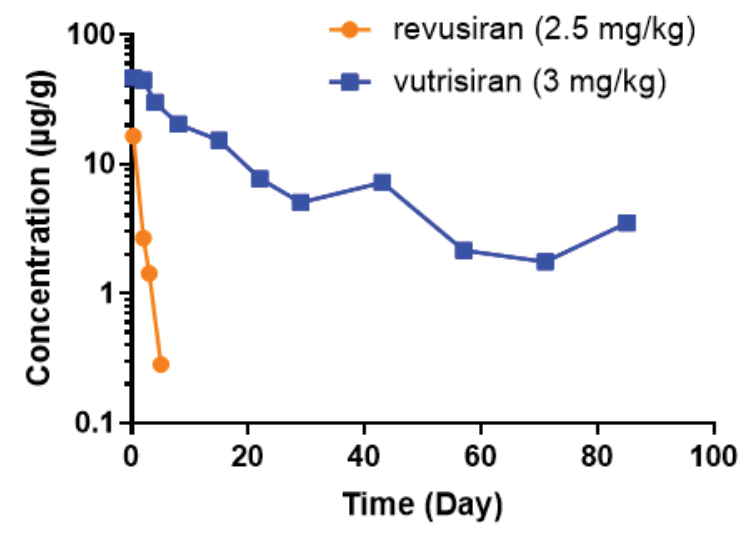

C.

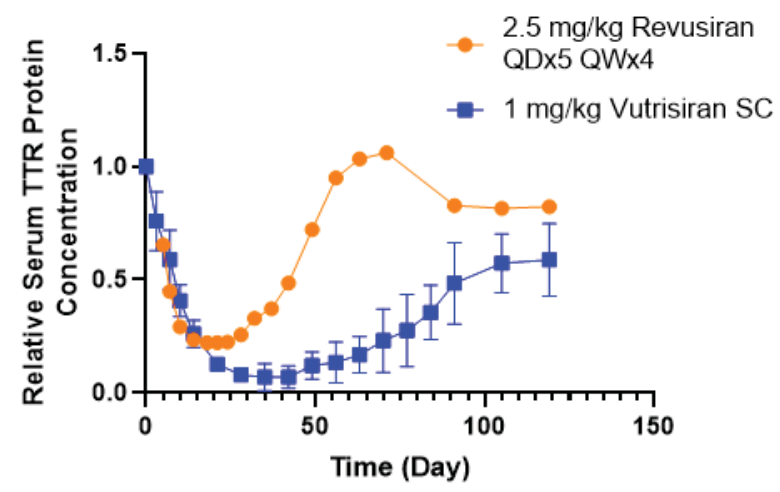

B.

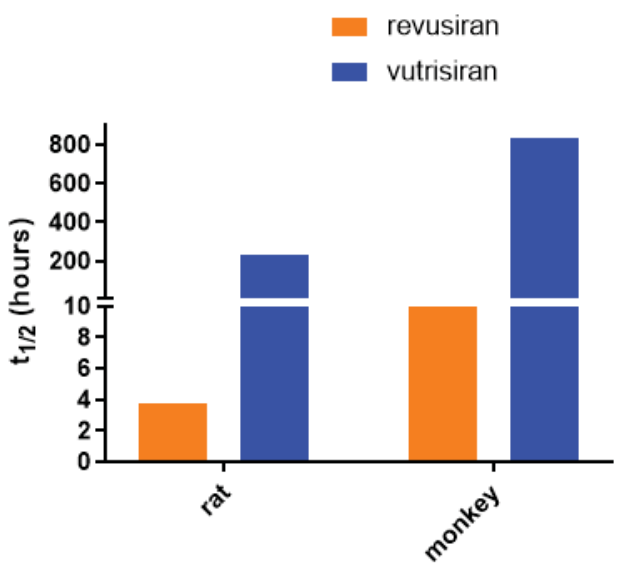

D.

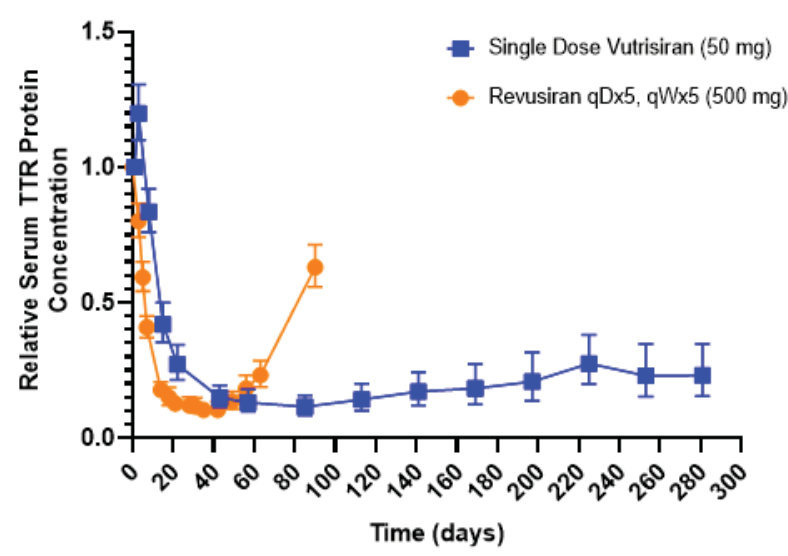

Figure 10 